\title{
Production and backreaction of fermions from axion-SU(2) gauge fields during inflation
}

\author{
Leila Mirzagholi@, ${ }^{*}$ Azadeh Maleknejad, ${ }^{\dagger}$ and Kaloian D. Lozanov $\odot^{*}$ \\ Max-Planck-Institute for Astrophysics, Karl-Schwarzschild-Straße 1, 85741 Garching, Germany
}

(Received 19 February 2020; accepted 17 March 2020; published 20 April 2020)

\begin{abstract}
$S U(2)$ gauge fields and axions can have a stable, isotropic and homogeneous configuration during inflation. However, couplings to other matter species lead to particle production, which in turn induces backreaction on and destabilization of the non-Abelian and axion background. In this paper, we first study the particle production by a $S U(2)$ gauge field coupled to a massive Dirac doublet. To carry out this calculation we have made two technical improvements compared to what has been done in the literature. First, we apply the antisymmetrization of the operators to treat particles and antiparticles on equal footing; second, to deal with the UV divergences, we apply instantaneous subtraction. We find that the backreaction of produced fermions on the $S U(2)$ background is negligible for model parameters of observational interest. Next, we consider production of fermions due to coupling to the axion. The tree-level backreaction on the gauge fields, as well as on the axion, is vanishingly small. We also provide an estimate for the loop effects.
\end{abstract}

DOI: 10.1103/PhysRevD.101.083528

\section{INTRODUCTION}

Coupled axion and $S U(2)$ gauge fields can develop nontrivial vacuum expectation values (VEVs) [1-4] during cosmic inflation [5-8]. These inflationary models have a rich phenomenology that is not shared by canonical single scalar field inflation models (see [9] for a review). As was first discovered by one of the authors (A. M.), when the conformal symmetry of Yang-Mills theory is broken by an effective $(F \tilde{F})^{2}$ term in the Lagrangian, non-Abelian gauge fields acquire an isotropic and homogeneous background VEV solution during inflation [1,2]. Since then, several inflationary models with the $S U(2) \mathrm{VEV}$ have been introduced and studied which share the above features $[3,4,10-13]$.

This background gauge field can provide a source for particle production during inflation. Despite being energetically subdominant, the axion- $S U(2)$ gauge field can produce potentially detectable signals during inflation. It can generate stochastic backgrounds of chiral gravitational waves $[10,12,14-16]$, tensor non-Gaussianity

\footnotetext{
*1mirz@MPA-Garching.MPG.DE

†amalek@MPA-Garching.MPG.DE

*klozanov@MPA-Garching.MPG.DE
}

Published by the American Physical Society under the terms of the Creative Commons Attribution 4.0 International license. Further distribution of this work must maintain attribution to the author(s) and the published article's title, journal citation, and DOI. Funded by SCOAP ${ }^{3}$.
[17-19] and the observed matter asymmetry in the Universe [20-23]. ${ }^{1}$

The upcoming LiteBIRD [26,27] and CMB Stage-4 experiments are set to provide further constraints on the axion-gauge fields [28-30]. That is why it is important to check if models are viable phenomenologically. In particular, it is critical to see if couplings to other matter species can give rise to particle production and backreaction on the axion-gauge field background, thereby affecting the sourcing of observational signals. In [31], a charged scalar field was coupled to the $S U(2)$ gauge field, and production of pairs of charged particles in the nontrivial gauge field background (known as the Schwinger effect [32]) was studied in de Sitter spacetime. It was found that the backreaction of the scalar particles on the $S U(2)$ background is negligible in the viable parameter regions of the simplest axion-SU(2) models. In [33], the backreaction of the extra spin-2 field in this setup was analytically studied for all the inflationary models involving the $S U(2)$ gauge field.

In this paper we continue our investigation of particle production by the axion-SU(2) gauge field during inflation. This time, we study coupling of the $S U(2)$ gauge field to a pair of massive Dirac fermions, $i \bar{\Psi} \not \supset \Psi$. We also add an interaction between axion and the axial fermionic current, $J^{\mu 5} \partial_{\mu} \varphi / \Lambda$, which is naturally expected in this type of models. We then calculate the backreaction of the fermions on the $S U(2)$ and axion background dynamics during

\footnotetext{
${ }^{1}$ For a study of the nonlinear impact of the spectator sector on the comoving curvature perturbation during inflation see [24,25].
} 
inflation, following the framework we have established in $[31,33]$.

Fermionic particle production in de Sitter spacetime and its backreaction implications have been studied in the context of $U(1)$ theories. The case of a nontrivial Abelian gauge field background without an axion was studied in [34]; a slowly evolving axion background with no gauge field interactions was studied in [35,36]; and a combination of the two, assuming a massless fermion, was studied in [37].

As for the $S U(2)$ gauge field background, the simplest fermionic non-Abelian model was studied in the recent work [38], where a massless doublet of Dirac fields is coupled covariantly to the $S U(2)$ gauge field. The main aim of [38] is to describe the fermionic particle production due to the quantum (loop) effects from the Adler-Bell-Jackiw (or chiral) anomaly. Our work not only extends their study to models with massive fermions and coupling to the axion background, but also provides the first detailed analysis of the allowed parameter space by cosmological and backreaction constraints.

We find important differences between the Abelian and non-Abelian models. Most notably, the leading order fermionic backreaction on the $S U(2)$ background is significantly smaller than in the fermionic Abelian [34] (as well as in the scalar Abelian [39-43]) cases. We find that the tree-level backreaction on the axion field background vanishes, unlike in $U(1)$ theories [35-37]. We thus conclude that, the inflationary scenarios involving an axion$S U(2)$ gauge field spectator sector remain healthy and unaffected when couplings to gauged fermions are present.

The organization of the paper is the following. In Sec. II we introduce our model. Section III deals with the evolution and production of fermions in the time-dependent axion-SU(2) background in a de Sitter universe. The results for the induced fermionic backreaction are presented in Sec. IV. Section V is devoted to discussions and concluding remarks. Most of the technical details, including lengthy derivations, notations and conventions are delegated to the Appendixes.

\section{FERMIONS IN AXION-SU(2) GAUGE FIELD INFLATION}

In this work, we study the fermion production by a slowly evolving homogeneous and isotropic $S U(2)$ gauge field during inflation. The class of inflationary models involving such $S U(2)$ VEV has been first introduced in $[1,2]$. Since then several different realizations of this class of models have been introduced and studied, e.g., [3,4]. See Sec. 2 of [33] and the references therein for a recent review on the models so far in the literature.

We assume slow-roll inflation with the background FLRW metric

$$
d s^{2}=a^{2}(\tau)\left(-d \tau^{2}+\delta_{i j} d x^{i} d x^{j}\right),
$$

where $\tau$ is the conformal time, and the scale factor, $a(\tau)$, is related to the Hubble parameter, $H$, as

$$
a(\tau) \simeq-\frac{1}{H \tau} \quad \text { and } \quad H \simeq \text { const }
$$

Besides, we have a homogeneous and isotropic $S U(2)$ gauge field background generated by one of the possible realizations of this class of models. In the temporal gauge $\left(\mathbf{A}_{0}=0\right)$, we have in $[1,2]$

$$
\mathbf{A}_{i}=A_{i}^{a} \mathbf{T}_{a}=a(\tau) \psi(\tau) \delta_{i}^{a} \mathbf{T}_{a},
$$

where $\psi(\tau) \simeq$ const during slow-roll inflation and $\mathbf{T}_{a}$ are the generators of the $S U(2)$ group

$$
\left[\mathbf{T}_{a}, \mathbf{T}_{b}\right]=i \epsilon^{c}{ }_{a b} \mathbf{T}_{c} .
$$

Therefore, the gauge field has an almost constant energy density during inflation.

To avoid clutter, we suppress the spacetime indices of the Dirac matrices and spinors, unless otherwise stated. For example, $\gamma^{0}$ is a $4 \times 4$ matrix, which can act on the fourdimensional column spinor $\Psi^{1}$ or can be acted upon by the four-dimensional row spinor $\bar{\Psi}^{1} \equiv \Psi^{1 \dagger} \gamma^{0}$. We will have to deal with eight-, four- and two-component spinors which are acted upon by $8 \times 8,4 \times 4$ and $2 \times 2$ matrices, respectively to this end, we adopt the following notation. If the spinor (or the matrix) is eight (or $8 \times 8$ ) dimensional, then it has a tilde $(\sim)$ on top. The notation for the four-dimensional spinor and matrix remains unaltered, whereas the two-dimensional ones are written in boldface. Finally, $\mathrm{I}_{n}$ represents the $n \times n$ identity matrix and the gamma matrices are in the Dirac representation unless otherwise stated.

We consider a charged doublet of Dirac fermions,

$$
\tilde{\Psi}=\left(\begin{array}{c}
\Psi^{1} \\
\Psi^{2}
\end{array}\right)
$$

with the free theory

$$
S_{\text {fermion }}=\int \mathrm{d}^{4} x \sqrt{-g}[i \overline{\tilde{\Psi}} \not D \tilde{\Psi}-m \overline{\tilde{\Psi}} \tilde{\Psi}],
$$

where $\overline{\tilde{\Psi}}=\left(\begin{array}{ll}\bar{\Psi}_{1} & \bar{\Psi}_{2}\end{array}\right)$, and $\not D$ is

$$
\not D \equiv D_{\mu} \otimes \gamma^{\mu}=\mathbf{e}_{\alpha}^{\mu}\left[\mathbf{I}_{2} \nabla_{\mu}-i g_{A} A_{\mu}^{a} \mathbf{T}_{a}\right] \otimes \gamma^{\alpha},
$$

in which $\otimes$ is the Kronecker product, $\mathbf{e}^{\mu}{ }_{\alpha}=a^{-1}(\tau) \delta_{\alpha}^{\mu}$ are the vierbeins given by $g^{\mu \nu}=\mathbf{e}^{\mu}{ }_{\alpha} \mathbf{e}^{\nu}{ }_{\beta} \eta^{\alpha \beta}$, and $\gamma^{\alpha}$ are the flat space Dirac matrices

$$
\left\{\gamma^{\alpha}, \gamma^{\beta}\right\}=2 \eta^{\alpha \beta} \mathrm{I}_{4}
$$


See Appendix A for details. Notice that $\gamma^{\mu}=\mathbf{e}_{\alpha}^{\mu} \gamma^{\alpha}=$ $a(\tau) \delta_{\alpha}^{\mu} \gamma^{\alpha}$, where $\alpha$ and $\beta$ represent the local Lorentz indices while $\mu$ and $\nu$ represent spacetime coordinates.

Since the inflationary setups in this paper involve axion fields, the fermionic sector can have the following effective interaction with the axion (see for instance [44]):

$$
S_{\text {int }}=\int \mathrm{d}^{4} x \sqrt{-g}\left[\beta \frac{\lambda \varphi}{f} \nabla_{\mu} J_{5}^{\mu}\right],
$$

where $\varphi$ is the axion field, $f$ is the axion decay constant, $\lambda$ is the dimensionless coefficient of the Chern-Simons interaction term of the axion, and $\beta$ is a dimensionless parameter. The quantity $\beta$ can be of order unity. Here, $J_{5}^{\mu}$ is the fermionic chiral current given as

$$
J_{5}^{\mu} \equiv \overline{\tilde{\Psi}} \mathbf{I}_{2} \otimes\left(\gamma^{\mu} \gamma^{5}\right) \tilde{\Psi}
$$

where $\gamma^{5}=i \gamma^{0} \gamma^{1} \gamma^{2} \gamma^{3}$.

In summary, the fermion theory in an $S U(2)$-axion background given in (3) and $\varphi=\varphi(\tau)$ is specified by

$$
\begin{aligned}
S= & \int a^{4} \mathrm{~d} \tau \mathrm{d} x^{3} \frac{i}{a} \overline{\tilde{\Psi}}\left[\mathbf{I}_{2}\left(\partial_{\tau}+\frac{3}{2} \mathcal{H}\right) \otimes \gamma^{0}\right. \\
& +\delta_{\alpha}^{i}\left(\mathbf{I}_{2} \partial_{i}-i a g_{A} \psi \delta_{i}^{a} \mathbf{T}_{a}\right) \otimes \gamma^{\alpha} \\
& \left.+i a m \mathbf{I}_{8}+\beta \frac{i \lambda \partial_{\tau} \varphi}{f} \mathbf{I}_{2} \otimes\left(\gamma^{0} \gamma^{5}\right)\right] \tilde{\Psi},
\end{aligned}
$$

where the second term in the right-hand side comes from the spin connection [see (A4) and (A8)]. As implied by the above action, the canonically normalized field is

$$
\tilde{\psi} \equiv a^{\frac{3}{2}} \tilde{\Psi}
$$

Using $\mathbf{T}_{a}=\frac{1}{2} \boldsymbol{\sigma}^{i} \delta_{i}^{a}$ ( $\boldsymbol{\sigma}^{i}$ are the Pauli matrices) in (11), we find

$$
\begin{aligned}
\mathcal{L}= & i \overline{\tilde{\psi}}\left[\mathbf{I}_{2} \partial_{\tau} \otimes \gamma^{0}+\left(\mathbf{I}_{2} \partial_{i}-\frac{i}{2} \xi_{A} \mathcal{H} \boldsymbol{\sigma}^{i}\right) \otimes \gamma^{i}\right. \\
& \left.+i \mu_{\mathrm{m}} \mathcal{H} \mathrm{I}_{8}+2 i \xi_{\varphi} \mathcal{H} \mathbf{I}_{2} \otimes\left(\gamma^{0} \gamma^{5}\right)\right] \tilde{\psi},
\end{aligned}
$$

where $\xi_{A}, \xi_{\varphi}$, and $\mu_{\mathrm{m}}$ are dimensionless parameters defined by

$$
\xi_{A} \equiv \frac{g_{A} \psi}{H}, \quad \xi_{\varphi} \equiv \beta \frac{\lambda \partial_{\tau} \varphi}{2 a f H}, \quad \mu_{\mathrm{m}} \equiv \frac{m}{H}
$$

For the sake of completeness, here we define another related dimensionless quantity in the axion inflation backgrounds

$$
\xi \equiv \frac{\lambda \partial_{\tau} \varphi}{2 a f H}
$$

which during slow-roll inflation is related to $\xi_{A}$ as $\xi \simeq \frac{1+\xi_{A}^{2}}{\xi_{A}}$ in the massless $S U(2)$ models. In our setup, $\xi$ and $\xi_{\varphi}$ are related as

$$
\xi_{\varphi}=\beta \xi,
$$

where $\beta$ is of order unity.

Up to this point, we wrote the theory in the flavor frame in terms of an 8-spinor in real space. However, in Fourier space, the setup is reducible into two irreducible 4-spinor subsectors in the helicity representation. Therefore, it is more convenient to go to Fourier space and write it in the extended helicity frame which we introduce now.

In Fourier space, we expand $\tilde{\psi}$ as

$$
\tilde{\psi}(\tau, \mathbf{x})=\int \mathrm{d}^{3} k e^{i \mathbf{k} \cdot \mathbf{x}} \tilde{\psi}_{\mathbf{k}} .
$$

For a given momentum, $\mathbf{k}$, the $8 \times 8$ helicity projection operators are

$$
\tilde{P}_{ \pm}(\mathbf{k})=\mathbf{I}_{2} \otimes\left(\frac{\mathrm{I}_{4} \pm k^{i} \gamma^{i}}{2}\right)
$$

which decompose $\tilde{\psi}_{\mathbf{k}}$ into the plus and minus helicity states as

$$
\tilde{\psi}_{\mathbf{k}}^{ \pm}=\tilde{P}_{ \pm}(\mathbf{k}) \tilde{\psi}_{\mathbf{k}},
$$

where $\tilde{\psi}_{\mathbf{k}}=\tilde{\psi}_{\mathbf{k}}^{+}+\tilde{\psi}_{\mathbf{k}}^{-}$. The helicity representation decomposes the system of 8-spinor in real space in Eq. (13) into two subspaces of 4-spinors in real space

$$
\tilde{\psi}=\psi^{+} \oplus \psi^{-}=\left(\begin{array}{l}
\psi^{+} \\
\psi^{-}
\end{array}\right),
$$

such that the theory is given as

$$
S[\tilde{\psi}]=S_{+}\left[\psi^{+}\right]+S_{-}\left[\psi^{-}\right] .
$$

We present the details of our consideration in Appendix B. Here, we write the final theories for each of the 4-spinor subspaces in Fourier space ${ }^{2}$

\footnotetext{
${ }^{2}$ Note that the form of the $S_{+}$is very similar to the action describing a single fermion derivatively coupled to an axion considered in [36]. There the authors make a local chiral transformation of the fermion basis to avoid unphysical behavior of the Bogoliubov coefficients in the massless fermion. However, for sufficiently large masses $\left(\mu_{m} \gtrsim 1\right)$ the transformation is not necessary. We do not follow this prescription for two reasons. First, the focus of the current study is limited to $\left(\mu_{m} \gtrsim 1\right)$. Second, the transformation is not applicable in the presence of our non-Abelian gauge field. In particular, it does not simplify the $S_{-}$action, because of the last term in the action.
} 


$$
\begin{aligned}
S_{+}= & \int \mathrm{d} \tau \mathrm{d}^{3} k \bar{\psi}_{\mathbf{k}}^{+}\left[i \gamma^{0} \partial_{\tau}-\gamma^{3} k-\left(2 \xi_{\varphi}-\frac{\xi_{A}}{2}\right) \mathcal{H} \lambda_{4}\right. \\
& \left.-\mu_{\mathrm{m}} \mathcal{H} \mathrm{I}_{4}\right] \psi_{\mathbf{k}}^{+}, \\
S_{-}= & \int \mathrm{d} \tau \mathrm{d}^{3} k \bar{\psi}_{\mathbf{k}}^{-}\left[i \gamma^{0} \partial_{\tau}+\gamma^{3} k-\left(2 \xi_{\varphi}+\frac{\xi_{A}}{2}\right) \mathcal{H} \lambda_{4}\right. \\
& \left.-\mu_{\mathrm{m}} \mathcal{H} \mathrm{I}_{4}+\gamma^{1} \xi_{A} \mathcal{H}\right] \psi_{\mathbf{k}}^{-},
\end{aligned}
$$

where

$$
\gamma^{0}=\left(\begin{array}{cc}
\mathbf{I}_{2} & \mathbf{0} \\
\mathbf{0} & -\mathbf{I}_{2}
\end{array}\right), \quad \gamma^{i}=\left(\begin{array}{cc}
\mathbf{0} & \boldsymbol{\sigma}^{i} \\
-\boldsymbol{\sigma}^{i} & \mathbf{0}
\end{array}\right), \quad \lambda_{4}=\left(\begin{array}{cc}
\mathbf{0} & \mathbf{I}_{2} \\
-\mathbf{I}_{2} & \mathbf{0}
\end{array}\right) .
$$

The field equations of $\psi_{\mathbf{k}}^{+}$and $\psi_{\mathbf{k}}^{-}$are

$$
\left[i \gamma^{0} \partial_{\tau}-\gamma^{3} k-\left(2 \xi_{\varphi}-\frac{\xi_{A}}{2}\right) \mathcal{H} \lambda_{4}-\mu_{\mathrm{m}} \mathcal{H} \mathrm{I}_{4}\right] \psi_{k}^{+}=0
$$

and

$$
\left[i \gamma^{0} \partial_{\tau}+\gamma^{3} k-\left(2 \xi_{\varphi}+\frac{\xi_{A}}{2}\right) \mathcal{H} \lambda_{4}-\mu_{\mathrm{m}} \mathcal{H} \mathrm{I}_{4}+\gamma^{1} \xi_{A} \mathcal{H}\right] \psi_{\mathbf{k}}^{-}=0
$$

respectively. Therefore, we have two independent Dirac fermions, $\psi_{\mathbf{k}}^{ \pm}$. We solve them in the next section. Before that, let us take a closer look at the field equations to have a qualitative understanding of each field.

Our Dirac fields can be expanded as

$$
\psi_{\mathbf{k}}^{ \pm}=\sum_{s= \pm}\left(\begin{array}{c}
\psi_{s}^{ \pm \uparrow}(\tau, k) \mathbf{E}_{s} \\
s \psi_{s}^{ \pm \downarrow}(\tau, k) \mathbf{E}_{s}
\end{array}\right),
$$

where $\psi_{s}^{ \pm \uparrow}(\tau, k)$ and $\psi_{s}^{ \pm \downarrow}(\tau, k)$ are mode functions and $\mathbf{E}_{s}$ with $s= \pm 1$ are the two-spinor polarization states

$$
\mathbf{E}_{+}=\left(\begin{array}{l}
1 \\
0
\end{array}\right) \quad \text { and } \quad \mathbf{E}_{-}=\left(\begin{array}{l}
0 \\
1
\end{array}\right)
$$

Since we are already in the helicity states of the given momentum $\mathbf{k}$, the 2-spinor polarization states are $k$ independent. Using the above in the field equations (24) and (25), we find the following.

(i) For the plus spinor field, the field is decoupled in terms of the polarization spinor $\mathbf{E}_{s}$. Thus, we have two pairs of coupled field equations for each polarization.

(ii) For the minus spinor field, the field equation is not diagonalizable in terms of $\mathbf{E}_{s}$. That is because of the extra (time-dependent) term proportional to $\gamma^{1}$ in the minus field equation (25). As a result, we have four coupled field equations. In the limit of either being well inside the horizon, i.e., $k \gg \mathcal{H}$, or $\xi_{A} \ll 1$, this term is negligible and the system reduces to two pairs of coupled field equations.

\section{FERMION PRODUCTION}

We now calculate the evolution of the plus and minus fermionic fields, $\psi_{k}^{ \pm}$. Since these 4 -spinors are decoupled [see Eqs. (24) and (25)], we consider them separately.

\section{A. $\psi^{+}$spinors}

We begin with the $\psi_{k}^{+}$modes, described by the $S_{+}$action given in Eq. (21). Since the modes are four-dimensional objects, their first order in time linear equation of motion, Eq. (24), should yield four linearly independent 4-spinor solutions, i.e.,

$$
\psi_{\boldsymbol{k}}^{+}=\sum_{s= \pm}\left[U_{s, \boldsymbol{k}}^{+}(\tau) a_{s, \boldsymbol{k}}^{+}+V_{s,-\boldsymbol{k}}^{+}(\tau) b_{s,-\boldsymbol{k}}^{+\dagger}\right]
$$

where creation and annihilation operators satisfy

$$
\begin{aligned}
& \left\{a_{s, \boldsymbol{k}}^{+}, a_{s^{\prime}, \boldsymbol{k}^{\prime}}^{+\dagger}\right\}=\delta_{s s^{\prime}} \delta^{(3)}\left(\boldsymbol{k}-\boldsymbol{k}^{\prime}\right), \\
& \left\{b_{s, \boldsymbol{k}}^{+}, b_{s^{\prime}, \boldsymbol{k}^{\prime}}^{+\dagger}\right\}=\delta_{s s^{\prime}} \delta^{(3)}\left(\boldsymbol{k}-\boldsymbol{k}^{\prime}\right) .
\end{aligned}
$$

We then decompose $U_{s, k}^{+}$and $V_{s, k}^{+}$as

$$
U_{s, \boldsymbol{k}}^{+}(\tau)=\frac{1}{\sqrt{2}}\left(\begin{array}{c}
\mathbf{E}_{s} u_{s}^{\uparrow}(k, \tau) \\
s \mathbf{E}_{s} u_{s}^{\downarrow}(k, \tau)
\end{array}\right),
$$

and

$$
V_{s,-k}^{+}(\tau)=\frac{1}{\sqrt{2}}\left(\begin{array}{c}
\mathbf{E}_{s} v_{s}^{\uparrow}(k, \tau) \\
s \mathbf{E}_{s} v_{s}^{\downarrow}(k, \tau)
\end{array}\right),
$$

where $\mathbf{E}_{s}$ are the 2-spinor polarization states defined in (27).

Using the procedure in Appendix $\mathrm{C}$ we can derive the initial conditions,

$u_{s}^{\uparrow}(k, \tau)=v_{s}^{\uparrow *}(k, \tau) \quad$ and $\quad u_{s}^{\downarrow}(k, \tau)=-v_{s}^{\downarrow *}(k, \tau)$.

Since $u_{s}^{\uparrow \downarrow}$ and $v_{s}^{\uparrow \downarrow}$ depend on each other, we can first solve the field equations of $u_{s}^{\uparrow \downarrow}$, and then use (32) to find $v_{s}^{\uparrow \downarrow}$ and read $V_{s,-\mathbf{k}}^{+}(\tau)$ as

$$
V_{s,-\mathbf{k}}^{+}(\tau)=\frac{1}{\sqrt{2}}\left(\begin{array}{c}
\mathbf{E}_{s} u_{s}^{\uparrow *}(k, \tau) \\
-s \mathbf{E}_{s} u_{s}^{\downarrow *}(k, \tau)
\end{array}\right) .
$$


Upon substituting the ansatz (28) and (30) into the field equation (24), we arrive at two sets of coupled field equations for each polarization:

$$
\begin{aligned}
& \left(i \partial_{\tau}-\mu_{\mathrm{m}} \mathcal{H}\right) u_{s}^{\uparrow}-\left[k+s\left(2 \xi_{\varphi}-\frac{\xi_{A}}{2}\right) \mathcal{H}\right] u_{s}^{\downarrow}=0, \\
& \left(i \partial_{\tau}+\mu_{\mathrm{m}} \mathcal{H}\right) u_{s}^{\downarrow}-\left[k+s\left(2 \xi_{\varphi}-\frac{\xi_{A}}{2}\right) \mathcal{H}\right] u_{s}^{\uparrow}=0 .
\end{aligned}
$$

To find analytical solutions we make the following decomposition:

$u_{s}^{\uparrow}=\frac{1}{\sqrt{2 \tilde{\tau}}}\left(Y_{s}+Z_{s}\right)$ and $\quad u_{s}^{\downarrow}=\frac{1}{\sqrt{2 \tilde{\tau}}}\left(Y_{s}-Z_{s}\right)$,

where $\tilde{\tau}$ is the physical momentum rescaled by $H$, i.e.,

$$
\tilde{\tau} \equiv \frac{k}{a H}=-k \tau .
$$

The coupled set of first order differential equations (34) and (35) can be decoupled into two second order differential equations for $Y_{s}$ and $Z_{s}$ as

$$
\begin{aligned}
& \partial_{\tilde{\tau}}^{2} Y_{s}+\left[1-\frac{2 i \kappa_{s}^{+}}{\tilde{\tau}}+\frac{1 / 4-\mu^{+2}}{\tilde{\tau}^{2}}\right] Y_{s}=0, \\
& \partial_{\tilde{\tau}}^{2} Z_{s}+\left[1-\frac{2 i \tilde{\kappa}_{s}^{+}}{\tilde{\tau}}+\frac{1 / 4-\mu^{+2}}{\tilde{\tau}^{2}}\right] Z_{s}=0,
\end{aligned}
$$

where $\kappa_{s}^{+}$, and $\tilde{\kappa}_{s}^{+}$are

$$
\kappa_{s}^{+}=\frac{1}{2}+i s\left(2 \xi_{\varphi}-\frac{\xi_{A}}{2}\right)
$$

and

$$
\tilde{\kappa}_{s}^{+}=-\frac{1}{2}+i s\left(2 \xi_{\varphi}-\frac{\xi_{A}}{2}\right)
$$

while $\mu^{+}$is

$$
\mu^{+}=i\left[\mu_{\mathrm{m}}^{2}+\left(2 \xi_{\varphi}-\frac{\xi_{A}}{2}\right)^{2}\right]^{\frac{1}{2}}
$$

The general solutions for Eqs. (38) and (39) are $W_{\kappa, \mu}(-2 i \tilde{\tau})$ and $M_{\kappa, \mu}(-2 i \tilde{\tau})$ Whittaker functions. Setting the BunchDavies (BD) vacuum as the initial condition for $u_{s}^{\uparrow}$ and $u_{s}^{\downarrow}$,

$$
\lim _{\tau \rightarrow-\infty} u_{s}^{\uparrow}(\tau, k)=\frac{1}{(2 \pi)^{\frac{3}{2}}} \frac{e^{-i k \tau}}{\sqrt{2 k}},
$$

and

$$
\lim _{\tau \rightarrow-\infty} u_{s}^{\downarrow}(\tau, k)=\frac{1}{(2 \pi)^{\frac{3}{2}}} \frac{e^{-i k \tau}}{\sqrt{2 k}}
$$

and using the asymptotic form of the $W$ and $M$ functions in (A9), we find that $Y_{s}$ and $Z_{s}$ are given by

$$
Y_{s}=b_{1 s} W_{\kappa_{s}^{+}, \mu^{+}}(-2 i \tilde{\tau}),
$$

and

$$
Z_{s}=b_{2 s} W_{\tilde{\kappa}_{s}^{+}, \mu^{+}}(-2 i \tilde{\tau}) .
$$

Therefore, in the asymptotic past limit, we have $Z_{s}=\frac{i}{2}\left(b_{2 s} / b_{1 s}\right) \tilde{\tau}^{-1} Y_{s} \ll Y_{s}$. Combination of the BunchDavies vacuum condition in (43) and the asymptotic form of the $W$ function in (A9) gives $b_{1 s}=\frac{1}{(2 \pi)^{\frac{3}{2}}} e^{s\left(\xi_{A} / 4-\xi_{\varphi}\right) \pi}$. Moreover, subtracting (35) from (34) and keeping the dominant terms in the asymptotic past limit, we find $b_{2 s}=-i \mu_{\mathrm{m}} b_{1 s}$. Finally, we have

$$
\begin{aligned}
Y_{s} & =\frac{1}{(2 \pi)^{\frac{3}{2}}} e^{s\left(\xi_{A} / 4-\xi_{\varphi}\right) \pi} W_{\kappa_{s}^{+}, \mu^{+}}(-2 i \tilde{\tau}), \\
Z_{s} & =-\frac{i \mu_{\mathrm{m}}}{(2 \pi)^{\frac{3}{2}}} e^{s\left(\xi_{A} / 4-\xi_{\varphi}\right) \pi} W_{\tilde{\kappa}_{s}^{+}, \mu^{+}}(-2 i \tilde{\tau}) .
\end{aligned}
$$

Note that the amplitudes and the relative phases of the Bunch-Davies vacuum modes are such that the corresponding Hamiltonian is diagonalized. We present the detailed calculation of the Hamiltonian in Appendix C. We note that the Hamiltonian diagonalization leaves a residual freedom in choosing the initial conditions for the mode functions. The same applies to the computation in the next section. In both cases, we chose a set of initial conditions for which the Hamiltonian is diagonalized in the subhorizon limit.

\section{B. $\psi^{-}$spinors}

Next, we calculate the $\psi_{\boldsymbol{k}}^{-}$modes, described by the $S_{-}$action given in Eq. (22). Since the modes are fourdimensional objects, their first order in time linear equation of motion, Eq. (25), should yield four linearly independent 4-spinor solutions, i.e.,

$$
\psi_{\boldsymbol{k}}^{-}=\sum_{s= \pm}\left[U_{s, \boldsymbol{k}}^{-}(\tau) a_{s, \boldsymbol{k}}^{-}+V_{s,-\boldsymbol{k}}^{-}(\tau) b_{s,-\boldsymbol{k}}^{-\dagger}\right],
$$

where creation and annihilation operators satisfy

$$
\begin{aligned}
& \left\{a_{s, k}^{-}, a_{s^{\prime}, k^{\prime}}^{-\dagger}\right\}=\delta_{s s^{\prime}} \delta^{(3)}\left(\boldsymbol{k}-\boldsymbol{k}^{\prime}\right), \\
& \left\{b_{s, \boldsymbol{k}}^{-}, b_{s^{\prime}, \boldsymbol{k}^{\prime}}^{-\dagger}\right\}=\delta_{s s^{\prime}} \delta^{(3)}\left(\boldsymbol{k}-\boldsymbol{k}^{\prime}\right) .
\end{aligned}
$$

The Lagrangian of the minus subspace is not diagonalizable in a time-independent frame. Hence, the helicity eigenstates are only the eigenstates of the Lagrangian in the 
asymptotic past limit. Therefore, we adopt a more general trial vector solution:

$U_{s, k}^{-}(\tau)=\frac{1}{\sqrt{2}}\left(\begin{array}{c}\mathbf{E}_{s} u_{s,+}^{\uparrow}(k, \tau) \\ \mathbf{E}_{s} u_{s,+}^{\downarrow}(k, \tau)\end{array}\right)+\frac{1}{\sqrt{2}}\left(\begin{array}{c}\mathbf{E}_{-s} u_{s,-}^{\uparrow}(k, \tau) \\ -s \mathbf{E}_{-s} u_{s,-}^{\downarrow}(k, \tau)\end{array}\right)$,
$V_{s, k}^{-}(\tau)=\frac{1}{\sqrt{2}}\left(\begin{array}{c}\mathbf{E}_{s} v_{s,+}^{\uparrow}(k, \tau) \\ s \mathbf{E}_{s} v_{s,+}^{\downarrow}(k, \tau)\end{array}\right)+\frac{1}{\sqrt{2}}\left(\begin{array}{c}\mathbf{E}_{-s} v_{s,-}^{\uparrow}(k, \tau) \\ -s \mathbf{E}_{-s} v_{s,-}^{\downarrow}(k, \tau)\end{array}\right)$,

where $\mathbf{E}_{s}$ are the 2-spinor polarization states defined in (27). Since in the asymptotic past limit the system is diagonalized in this particular basis, we have

$$
\begin{aligned}
& \lim _{\tilde{\tau} \rightarrow \infty} u_{s,-}^{\uparrow}(k \tau)=\lim _{\tilde{\tau} \rightarrow \infty} u_{s,-}^{\downarrow}(k \tau)=0, \\
& \lim _{\tilde{\tau} \rightarrow \infty} v_{s,-}^{\uparrow}(k \tau)=\lim _{\tilde{\tau} \rightarrow \infty} v_{s,-}^{\downarrow}(k \tau)=0 .
\end{aligned}
$$

Using the procedure in Appendix $\mathrm{C}$, one can derive the initial conditions,

$$
u_{s,+}^{\uparrow}(k, \tau)=v_{s,+}^{\uparrow *}(k, \tau), u_{s,+}^{\downarrow}(k, \tau)=-v_{s,+}^{\downarrow *}(k, \tau) .
$$

Since $u_{s, p}^{\uparrow \downarrow}$ and $v_{s, p}^{\uparrow \downarrow}$ depend on each other, we can first solve the field equations of $u_{s, p}^{\uparrow \downarrow}$, and then use (53) to read $v_{s, p}^{\uparrow \downarrow}$ from their corresponding $u_{s, p}^{\uparrow \downarrow}$.

After the substitution of the ansatz from Eq. (50) into the equation of motion (25), we arrive at

$$
\begin{aligned}
& \left(i \partial_{\tau}-\mu_{\mathrm{m}} \mathcal{H}\right) u_{s, p}^{\uparrow}+\left[k-s p\left(2 \xi_{\varphi}+\frac{\xi_{A}}{2}\right) \mathcal{H}\right] u_{s, p}^{\downarrow} \\
& \quad-s p \xi_{A} \mathcal{H} u_{s,-p}^{\downarrow}=0, \\
& \left(i \partial_{\tau}+\mu_{\mathrm{m}} \mathcal{H}\right) u_{s, p}^{\downarrow}+\left[k-s p\left(2 \xi_{\varphi}+\frac{\xi_{A}}{2}\right) \mathcal{H}\right] u_{s, p}^{\uparrow} \\
& \quad+\operatorname{sp} \xi_{A} \mathcal{H} u_{s,-p}^{\uparrow}=0 .
\end{aligned}
$$

Note also that for each given $s$ and $p$, we have a pair of coupled equations between $p=+$ and $p=-$ fields.

Following our approach from the previous section, we make the decomposition

$$
u_{s, p}^{\uparrow}=\frac{1}{\sqrt{2 \tilde{\tau}}}\left(Y_{s, p}+Z_{s, p}\right)
$$

and

$$
u_{s, p}^{\downarrow}=\frac{1}{\sqrt{2 \tilde{\tau}}}\left(Y_{s, p}-Z_{s, p}\right),
$$

yielding

$$
\begin{gathered}
i \partial_{\tilde{\tau}} Y_{s, p}-\left[1-\frac{s p\left(4 \xi_{\varphi}+\xi_{A}\right)-i}{2 \tilde{\tau}}\right] Y_{s, p} \\
+\frac{\mu_{\mathrm{m}}}{\tilde{\tau}} Z_{s, p}-\frac{s p \xi_{A}}{\tilde{\tau}} Z_{s,-p}=0, \\
i \partial_{\tilde{\tau}} Z_{s, p}+\left[1-\frac{s p\left(4 \xi_{\varphi}+\xi_{A}\right)+i}{2 \tilde{\tau}}\right] Z_{s, p} \\
+\frac{\mu_{\mathrm{m}}}{\tilde{\tau}} Y_{s, p}+\frac{s p \xi_{A}}{\tilde{\tau}} Y_{s,-p}=0 .
\end{gathered}
$$

We can reduce the above coupled first order equations to pairs of coupled second order equations:

$$
\begin{gathered}
\partial_{\tilde{\tau}}^{2} Y_{s, p}+\left[1+\frac{i-2 s p\left(\xi_{\varphi}+\xi_{A} / 2\right)}{\tilde{\tau}}\right. \\
\left.+\frac{1 / 4+\mu_{\mathrm{m}}^{2}+\left(\xi_{\varphi}+\xi_{A} / 2\right)^{2}+\xi_{A}^{2}}{\tilde{\tau}^{2}}\right] Y_{s, p} \\
-\frac{2 \xi_{A}\left(\xi_{\varphi}+\xi_{A} / 2\right)}{\tilde{\tau}^{2}} Z_{s,-p}=0, \\
\partial_{\tilde{\tau}}^{2} Z_{s,-p}+\left[1-\frac{i-2 s p\left(\xi_{\varphi}+\xi_{A} / 2\right)}{\tilde{\tau}}\right. \\
\left.+\frac{1 / 4+\mu_{\mathrm{m}}^{2}+\left(\xi_{\varphi}+\xi_{A} / 2\right)^{2}+\xi_{A}^{2}}{\tilde{\tau}^{2}}\right] Z_{s,-p} \\
-\frac{2 \xi_{A}\left(\xi_{\varphi}+\xi_{A} / 2\right)}{\tilde{\tau}^{2}} Y_{s, p}=0 .
\end{gathered}
$$

Unlike before, the system cannot be solved analytically. Therefore, we solve them numerically. Furthermore, the amplitudes and the phases of the modes are adjusted to diagonalize the Hamiltonian. For a detailed derivation of the Hamiltonian see Appendix C.

\section{BACKREACTIONS}

The action (6) has a Noether current associated to the $S U(2)$ isospin,

$$
J^{\mu a}=\delta_{\alpha}^{\mu} \frac{g_{A}}{2 a^{4}} \overline{\tilde{\psi}} \boldsymbol{\sigma}^{a} \otimes \gamma^{\alpha} \tilde{\psi},
$$

and the axial vector current, $J_{5}^{\mu}$, given in (10). Notice that $J^{\mu a} \equiv \frac{\delta S_{\text {fermion }}}{\sqrt{-g} \delta A_{\mu}^{a}}$ satisfies $\nabla_{\mu} J^{\mu a} \boldsymbol{\sigma}^{a}=\mathbf{0}$. The Noether current and divergence of the chiral current induce backreactions on the background field equations of an axion- $S U(2)$ setup. See [33] for details about a uniform presentation of the axion-SU(2) class of models and in particular its Sec. II for the background equations. The Noether 4-current backreacts on the background equation of the gauge field as

$$
\begin{gathered}
\partial_{\tau}^{2}(a \psi)+2 \mathcal{H} \partial_{\tau}(a \psi)+\left(\partial_{\tau} \mathcal{H}+\mathcal{H}^{2}\right)(a \psi) \\
+2 a^{3} g_{A}^{2} \psi^{3}-\frac{g_{A} \lambda}{f} a^{2} \partial_{\tau} \varphi \psi^{2}=-a^{2} \mathcal{J},
\end{gathered}
$$

where the spatially averaged component of the matter 3 -current is 


$$
\mathcal{J}=\frac{1}{3 a} \delta_{b}^{j}\left\langle J_{j}^{b}\right\rangle
$$

Moreover, the axial current backreacts on the axion background equation as

$\partial_{\tau}^{2} \varphi+2 \mathcal{H} \partial_{\tau} \varphi+a^{2} \partial_{\varphi} V+3 \frac{g_{A} \lambda}{f} a \psi^{2}\left(\mathcal{H} \psi+\partial_{\tau} \psi\right)=a^{2} \mathcal{B}$,

where the backreaction term is defined as

$$
\mathcal{B}=\beta \frac{\lambda}{2 f} \nabla_{\mu} J_{5}^{\mu}=-i m \beta \frac{\lambda}{a^{3} f} \overline{\tilde{\psi}} \gamma_{5} \tilde{\psi} .
$$

The last equality uses the field equations of the fermions.

We begin with an outline of our prescription for computing the VEVs of quadratic fermionic quantum operators such as $\mathcal{J}$ and $\mathcal{B}$. We then calculate the fermionic backreaction on the gauge field and the axion backgrounds.

\section{A. VEVs of quadratic fermionic operators}

In order to compute VEVs of quantum operators we follow $[45,46]$.

Consider a general four component fermionic field, similar to $\psi^{+}$and $\psi^{-}$defined in Sec. II.

$$
\begin{aligned}
\eta_{\alpha}(\boldsymbol{x}, \tau) & =\int \mathrm{d}^{3} k \eta_{\boldsymbol{k}, \alpha}(\tau) e^{i \boldsymbol{k} \cdot \boldsymbol{x}}, \\
\eta_{\mathbf{k}, \alpha}(\tau) & =\sum_{s= \pm}\left[U_{s, \boldsymbol{k}, \alpha}(\tau) c_{s, \boldsymbol{k}}+V_{s,-\boldsymbol{k}, \alpha}(\tau) d_{s,-\boldsymbol{k}}^{\dagger}\right]
\end{aligned}
$$

with a quadratic action

$$
S_{\eta}=\int d \tau \mathrm{d}^{3} k\left[i \eta_{k, \alpha}^{\dagger} \partial_{\tau} \eta_{k, \alpha}-\eta_{k, \alpha}^{\dagger} \Omega_{\alpha \beta}(k, \tau) \eta_{k, \beta}\right],
$$

and a Hamiltonian

$$
H_{\eta}=\int \mathrm{d}^{3} k \eta_{k, \alpha}^{\dagger} \Omega_{\alpha \beta}(k, \tau) \eta_{k, \beta},
$$

where $\alpha$ and $\beta$ run from 1 to 4 , and $c_{s, k}$ and $d_{s,-k}$ are timeindependent particle and antiparticle annihilation operators. Using Eq. (65) in the Hamiltonian, we obtain

$$
H_{\eta}=\int \mathrm{d}^{3} k\left(\mathbf{c}_{\boldsymbol{k}}^{\dagger}, \mathbf{d}_{-\boldsymbol{k}}\right)\left(\begin{array}{cc}
\mathcal{E}^{U} & \mathcal{F}^{\dagger} \\
\mathcal{F} & \mathcal{E}^{V}
\end{array}\right)\left(\begin{array}{c}
\mathbf{c}_{\boldsymbol{k}} \\
\mathbf{d}_{-\boldsymbol{k}}^{\dagger}
\end{array}\right),
$$

where

$$
\mathbf{c}_{\boldsymbol{k}}=\left[\begin{array}{ll}
c_{+, k} & c_{-, k}
\end{array}\right]^{T}, \quad \mathbf{d}_{\boldsymbol{k}}=\left[\begin{array}{ll}
d_{+, k} & d_{-, k}
\end{array}\right]^{T},
$$

and

$$
\mathcal{E}_{s s^{\prime}}^{U}=U_{s, k, \alpha}^{*} \Omega_{\alpha \beta} U_{s^{\prime}, \boldsymbol{k}, \beta}, \quad \mathcal{F}_{s s^{\prime}}=V_{s, \boldsymbol{k}, \alpha}^{*} \Omega_{\alpha \beta} V_{s^{\prime}, \boldsymbol{k}, \beta} .
$$

In the ground state of a given $k$-mode of the fermionic field, i.e., in the $\mathrm{BD}$ vacuum, the state vector is $\left|0_{\mathrm{BD}}\right\rangle$

$$
\mathbf{c}_{k, s}\left|0_{\mathrm{BD}}\right\rangle=0, \quad \mathbf{d}_{k, s}\left|0_{\mathrm{BD}}\right\rangle=0
$$

and

BD vacuum: $\mathcal{E}_{s s^{\prime}}^{U}=-\mathcal{E}_{s s^{\prime}}^{V}=\omega_{s} \delta_{s s^{\prime}}, \quad \mathcal{F}_{s s^{\prime}}=0$.

When the mode is excited, its $\mathcal{E}_{s s^{\prime}}^{U, V}$ can attain nonzero off-diagonal components and its $\mathcal{F}_{s s^{\prime}}$ can also become nonvanishing. However, we can still diagonalize $\mathcal{E}_{s s^{\prime}}^{U, V}$ with vanishing $\mathcal{F}_{s s^{\prime}}$ by rewriting the Hamiltonian as

$$
H_{\eta}=\int \mathrm{d}^{3} k\left(\check{\mathbf{c}}_{\boldsymbol{k}}^{\dagger}, \check{\mathbf{d}}_{-\boldsymbol{k}}\right)\left(\begin{array}{cc}
\check{\mathcal{E}}^{U} & \check{\mathcal{F}}^{\dagger} \\
\check{\mathcal{F}} & \check{\mathcal{E}}^{V}
\end{array}\right)\left(\begin{array}{c}
\check{\mathbf{c}}_{\boldsymbol{k}} \\
\check{\mathbf{d}}_{-\boldsymbol{k}}^{\dagger}
\end{array}\right),
$$

where

$$
\left(\begin{array}{c}
\check{\mathbf{c}}_{k}^{-}(\tau) \\
\check{\mathbf{d}}_{-k}^{-\dagger}(\tau)
\end{array}\right)=P_{k}(\tau)\left(\begin{array}{c}
\mathbf{c}_{k}^{-} \\
\mathbf{d}_{-k}^{-\dagger}
\end{array}\right)
$$

and

$$
P_{k}(\tau) P_{k}^{\dagger}(\tau)=\mathrm{I}_{4} .
$$

We choose the eigenvectors of

$$
\left(\begin{array}{ll}
\mathcal{E}^{U} & \mathcal{F}^{\dagger} \\
\mathcal{F} & \mathcal{E}^{V}
\end{array}\right)
$$

as the columns of $P_{k}^{\dagger}(\tau)$. Then,

$$
\check{\mathcal{E}}_{s s^{\prime}}^{U}=-\check{\mathcal{E}}_{s s^{\prime}}^{V}=\check{\omega}_{s} \delta_{s s^{\prime}}, \quad \check{\mathcal{F}}_{s s^{\prime}}=0,
$$

as promised.

After the transformation in Eq. (74), a new state vector has the properties of the physical vacuum, $\left|0_{\tau}\right\rangle$, defined as

$$
\check{\mathbf{c}}_{\boldsymbol{k}, s}\left|0_{\tau}\right\rangle=0, \quad \check{\mathbf{d}}_{\boldsymbol{k}, s}\left|0_{\tau}\right\rangle=0 .
$$

Note that we work in the Heisenberg picture in which the state vector is constant, i.e., it remains $\left|0_{\mathrm{BD}}\right\rangle$ throughout.

The fermionic field can be rewritten as

$$
\eta_{k, \alpha}(\tau)=\sum_{s= \pm}\left[\breve{U}_{s, k, \alpha}(\tau) \check{c}_{s, k}+\check{V}_{s,-k, \alpha}(\tau) \check{d}_{s,-\boldsymbol{k}}^{\dagger}\right]
$$

where

$$
\begin{aligned}
\check{U}_{s, \boldsymbol{k}, \alpha} & =\sum_{s^{\prime}= \pm}\left[U_{s^{\prime}, \boldsymbol{k}, \alpha} P_{\frac{3-s^{\prime}}{2}, \frac{3-s}{2}}^{\dagger}+V_{s^{\prime}-, \boldsymbol{k}, \alpha} P_{\frac{7-s^{\prime}}{2}, \frac{3-s}{2}}^{\dagger}\right], \\
\check{V}_{s,-\boldsymbol{k}, \alpha} & =\sum_{s^{\prime}= \pm}\left[V_{s^{\prime},-\boldsymbol{k}, \alpha} P_{\frac{7-s^{\prime}}{2}, \frac{7-s}{2}}^{\dagger}+U_{s^{\prime}, \boldsymbol{k}, \alpha} P_{\frac{3-s^{\prime}}{2}, \frac{7-s}{2}}^{\dagger}\right] .
\end{aligned}
$$


We wish to find the VEV of a Hermitian operator, which is quadratic in the fermionic field, of the general form

$\mathcal{O}(\mathbf{x}, \tau)=\int \mathrm{d}^{3} k \mathrm{~d}^{3} k^{\prime} e^{i\left(\boldsymbol{k}-\boldsymbol{k}^{\prime}\right) \cdot \mathbf{x}} \eta_{\boldsymbol{k}, \alpha}^{\dagger}(\tau) A_{\alpha, \beta}\left(\boldsymbol{k}, \boldsymbol{k}^{\prime}, \tau\right) \eta_{\boldsymbol{k}^{\prime}, \beta}(\tau)$,

with $A_{\alpha, \beta}^{\dagger}\left(\boldsymbol{k}, \boldsymbol{k}^{\prime}, \tau\right)=A_{\alpha, \beta}\left(\boldsymbol{k}^{\prime}, \boldsymbol{k}, \tau\right)$ by virtue of the Hermitian nature of the $\mathcal{O}$ operator.

When computing the VEV, we need to make sure that

(1) Particles and antiparticles are treated on equal footing.

(2) Only physical field excitations, i.e., those on top of the physical vacuum, contribute to the VEV.

To address the first point, we follow [45] and define the antisymmetrized operator,

$$
\begin{aligned}
\mathcal{O}_{a}(\mathbf{x}, \tau) \equiv & \int \mathrm{d}^{3} k \mathrm{~d}^{3} k^{\prime} e^{i\left(\boldsymbol{k}-\boldsymbol{k}^{\prime}\right) \cdot \boldsymbol{x}} A_{\alpha, \beta}\left(\boldsymbol{k}, \boldsymbol{k}^{\prime}, \tau\right)\left[\eta_{\boldsymbol{k}, \alpha}^{\dagger}(\tau), \eta_{\boldsymbol{k}^{\prime}, \beta}(\tau)\right] \\
= & \int \mathrm{d}^{3} k \mathrm{~d}^{3} k^{\prime} e^{i\left(\boldsymbol{k}-\boldsymbol{k}^{\prime}\right) \cdot \boldsymbol{x}} A_{\alpha, \beta}\left(\boldsymbol{k}, \boldsymbol{k}^{\prime}, \tau\right) \\
& \times \frac{1}{2}\left(\eta_{\boldsymbol{k}, \alpha}^{\dagger}(\tau) \eta_{\boldsymbol{k}^{\prime}, \beta}(\tau)-\eta_{\boldsymbol{k}^{\prime}, \beta}(\tau) \eta_{\boldsymbol{k}, \alpha}^{\dagger}(\tau)\right)
\end{aligned}
$$

which has the same classical counterpart as $\mathcal{O}$. The difference is that when we take the BD VEV, $\mathcal{O}$ receives only contributions from terms with $d_{s,-k} d_{s,-k}^{\dagger}$, i.e., the antiparticle creation and annihilation operators, whereas $\mathcal{O}_{a}$ receives contributions from both $c_{s, k} c_{s, \boldsymbol{k}}^{\dagger}$ and $d_{s,-\boldsymbol{k}} d_{s,-\boldsymbol{k}}^{\dagger}$.

To account for the second point, we follow [46] and we subtract from the BD VEV the expectation value with respect to $\left|0_{\tau}\right\rangle$. This way only nonvacuum field fluctuations contribute to the physical vacuum expectation value.

To sum up, the VEV of $\mathcal{O}$ is defined as

$\langle\mathcal{O}(\boldsymbol{x}, \tau)\rangle \equiv\left\langle 0_{\mathrm{BD}}\left|\mathcal{O}_{a}(\boldsymbol{x}, \tau)\right| 0_{\mathrm{BD}}\right\rangle-\left\langle 0_{\tau}\left|\mathcal{O}_{a}(\boldsymbol{x}, \tau)\right| 0_{\tau}\right\rangle$,

which reduces to

$$
\begin{aligned}
\langle\mathcal{O}(\boldsymbol{x}, \tau)\rangle= & \int \mathrm{d}^{3} k A_{\alpha \beta}(\boldsymbol{k}, \boldsymbol{k}, \tau) \sum_{s= \pm} \frac{1}{2} \\
& \times\left[\left(V_{s, \boldsymbol{k}, \alpha}^{*}(\tau) V_{s, \boldsymbol{k}, \beta}(\tau)-U_{s, \boldsymbol{k}, \beta}(\tau) U_{s, \boldsymbol{k}, \alpha}^{*}(\tau)\right)\right. \\
& \left.-\left(\check{V}_{s, \boldsymbol{k}, \alpha}^{*}(\tau) \check{V}_{s, \boldsymbol{k}, \beta}(\tau)-\check{U}_{s, \boldsymbol{k}, \beta}(\tau) \check{U}_{s, \boldsymbol{k}, \boldsymbol{\alpha}}^{*}(\tau)\right)\right] .
\end{aligned}
$$

Here, we are interested in the backreaction of the fermion on the gauge field equation, $\mathcal{J}^{ \pm}$, and the axion field equation, $\mathcal{B}^{ \pm}$, which can be computed by the above formula as

$$
\begin{aligned}
\left\langle\mathcal{J}^{s}\right\rangle= & \int \mathrm{d}^{3} k A_{\alpha \beta}^{\mathcal{J}^{s}}(\boldsymbol{k}, \boldsymbol{k}, \tau) \frac{1}{2} \\
& \times\left[\left(V_{s, \boldsymbol{k}, \alpha}^{*}(\tau) V_{s, \boldsymbol{k}, \beta}(\tau)-U_{s, \boldsymbol{k}, \beta}(\tau) U_{s, \boldsymbol{k}, \alpha}^{*}(\tau)\right)\right. \\
& \left.-\left(\check{V}_{s, \boldsymbol{k}, \alpha}^{*}(\tau) \check{V}_{s, \boldsymbol{k}, \beta}(\tau)-\check{U}_{s, \boldsymbol{k}, \beta}(\tau) \check{U}_{s, \boldsymbol{k}, \alpha}^{*}(\tau)\right)\right],
\end{aligned}
$$

and

$$
\begin{aligned}
\left\langle\mathcal{B}^{s}\right\rangle= & \int \mathrm{d}^{3} k A_{\alpha \beta}^{\mathcal{B}^{s}}(\boldsymbol{k}, \boldsymbol{k}, \tau) \frac{1}{2} \\
& \times\left[\left(V_{s, \boldsymbol{k}, \alpha}^{*}(\tau) V_{s, \boldsymbol{k}, \beta}(\tau)-U_{s, \boldsymbol{k}, \beta}(\tau) U_{s, \boldsymbol{k}, \alpha}^{*}(\tau)\right)\right. \\
& \left.-\left(\check{V}_{s, \boldsymbol{k}, \alpha}^{*}(\tau) \check{V}_{s, \boldsymbol{k}, \beta}(\tau)-\check{U}_{s, \boldsymbol{k}, \beta}(\tau) \check{U}_{s, \boldsymbol{k}, \alpha}^{*}(\tau)\right)\right],
\end{aligned}
$$

respectively.

\section{B. Backreaction on the $S U(2)$ background}

We now calculate the homogeneous and isotropic backreaction term on the $S U(2)$ background, $\mathcal{J}$, defined in Eq. (62). It conveniently separates into two independent contributions from the + and - fermions:

$$
\mathcal{J}=\mathcal{J}^{+}+\mathcal{J}^{-}
$$

The expressions for $\mathcal{J}^{+}$and $\mathcal{J}^{-}$take the form given in Eq. (85), with

$$
\begin{aligned}
A^{\mathcal{J}+}(\boldsymbol{k}, \boldsymbol{k}, \tau) & =\frac{g_{A}}{3 a^{3}}\left(\begin{array}{llll}
0 & 0 & 1 & 0 \\
0 & 0 & 0 & 1 \\
1 & 0 & 0 & 0 \\
0 & 1 & 0 & 0
\end{array}\right), \\
A^{\mathcal{J}-}(\boldsymbol{k}, \boldsymbol{k}, \tau) & =-\frac{g_{A}}{3 a^{3}}\left(\begin{array}{cccc}
0 & 0 & 1 & -2 \\
0 & 0 & -2 & 1 \\
1 & -2 & 0 & 0 \\
-2 & 1 & 0 & 0
\end{array}\right) .
\end{aligned}
$$

In Fig. 1, we show $\mathcal{J}^{+}$(left) and $\mathcal{J}^{-}$(right) for different values of the parameters $\xi_{\varphi}, m$ and $\xi_{A}$. We observe the following dependence:

(i) $\mathcal{J}^{+}$has a prominent dip when $2 \xi_{\varphi}-\xi_{A} / 2=0$, which occurs because the axion and gauge fieldinduced effective mass terms cancel. Besides this feature, for a fixed mass, $\mathcal{J}^{+}$increases monotonically with $\xi_{A}$. Otherwise when the "bare" mass of the fermion is the dominant scale, i.e., $m / H>\xi_{A}, \xi_{\varphi}$, we observe a decrease in particle production as the mass increases, as expected. In the opposite limit, $m / H<\xi_{A}, \xi_{\varphi}$, there is an increase in particle production as the mass increases, until the mass becomes the dominant scale. ${ }^{3}$

\footnotetext{
${ }^{3} \mathrm{~A}$ similar increase in particle production with the increase in mass was observed in [36]. In their setup, the fermion is not coupled to a gauge field but is derivatively coupled to an axion field.
} 

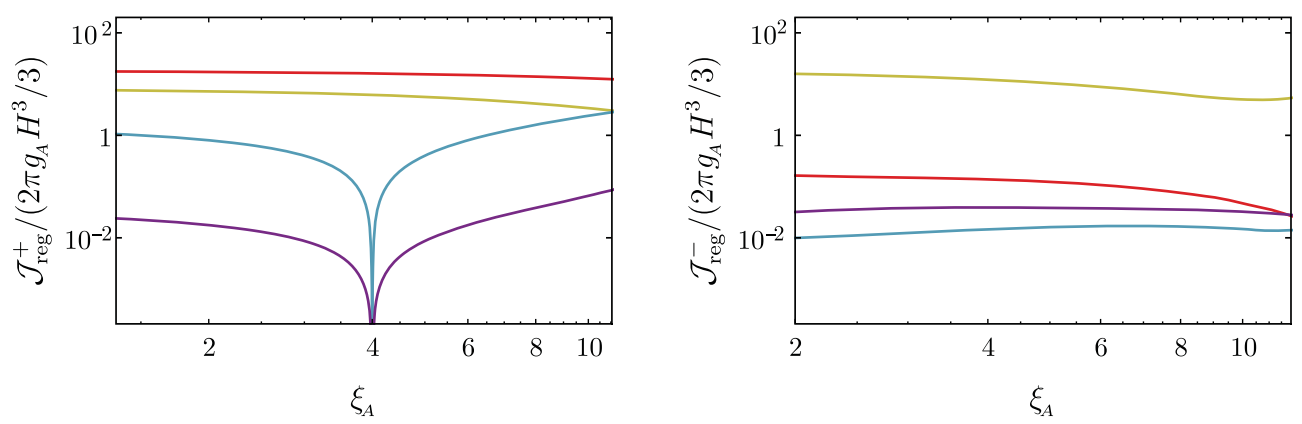

FIG. 1. The expectation values of the backreaction currents of the + (left panel) and - (right panel) fermions as a function of $\xi_{A}$ for $m=10 H, \xi_{\varphi}=1$ (purple); $m=H, \xi_{\varphi}=1$ (blue); $m=H, \xi_{\varphi}=10$ (gold); $m=10 H, \xi_{\varphi}=10$ (red). The prominent dip in the first panel at $2 \xi_{\varphi}-\xi_{A} / 2=0$ is due to an exact cancellation between the effective masses induced by the gauge field and the axion. Such a cancellation is not observed in the - fermions.

(ii) $\mathcal{J}^{-}$exhibits a complex behavior with the parameters which we attribute to the additional couplings in this sector. Using the current regularization scheme and for the parameter region of interest, $\mathcal{J}^{-}$never exceeds $\mathcal{J}^{+}$apart from the dips in $\mathcal{J}^{+}$, so the dominant contribution to the backreaction considered in the next section comes from $\mathcal{J}^{+}$.

(iii) When compared to the scalar case considered in [31], the fermion model has an important new feature. Unlike scalars, fermion particles are copiously produced as $\xi_{A}$ increases and dominate the other scales in the problem. Our setup provides a novel mechanism for efficient production of fermionic matter during inflation.

(iv) The fermion Schwinger particle production by a homogeneous $U(1)$ gauge field studied in [34] is different from our $S U(2)$ case with the isotropic and homogeneous VEV. In the $U(1)$ case the current decreases with the increase of the fermion mass. However, in both cases, the current increases like $\xi_{A}^{2}$ in the very strong gauge field limit.

Having computed $\mathcal{J}^{ \pm}$, we can compute their backreaction on the $S U(2)$ gauge field background, $\psi$, by following [33]. Assuming slow-roll evolution of the axion$S U(2)$, i.e., $\frac{\ddot{\psi}}{H^{2} \psi} \ll \frac{\dot{\psi}}{H \psi} \ll 1$, the field equation of the gauge field given in (61) can be approximated as

$$
3 H \dot{\psi}+\dot{H} \psi+V_{\mathrm{eff}, \psi}(\psi) \simeq 0,
$$

where a dot is a derivative with respect to cosmic time and the field derivative of the effective potential of $\psi$ is

$$
V_{\mathrm{eff}, \psi}(\psi) \simeq 2 H^{2} \psi\left(1+\xi_{A}^{2}\right)-\frac{g_{A} \lambda \dot{\varphi}}{f} \psi^{2} .
$$

Slow-roll demands $V_{\text {eff, } \psi}(\psi) \ll 1$, while each of the terms in the right-hand side can be much larger, e.g., $\frac{g_{A} \lambda \dot{\varphi}}{f} \psi^{2} / V_{\text {eff, } \psi} \gg 1$. On the other hand, $\mathcal{J}$ in the right-hand side of (61) should be at most on the order of the slow-roll suppressed terms, i.e.,

$$
\frac{\mathcal{J}}{H^{2} \psi} \ll 1
$$

so that it does not break the slow-roll dynamics in the background and can be considered as a perturbation correction. Otherwise, the perturbative expansion and slow-roll dynamics are not trustable and the models should be studied numerically.

Therefore, we define the regime of strong backreaction as

$$
\mathcal{J}<10^{-2} H^{2} \psi .
$$

We will use the above to explore the possible parameter space of one possible axion- $S U(2)$ gauge field model in Sec. IV D.

\section{Backreaction on the axion background}

We now turn to the homogeneous and isotropic backreaction term on the axion background, $\mathcal{B}$, defined in Eq. (64). It again splits into the sum of two independent + and - components:

$$
\mathcal{B}=\mathcal{B}^{+}+\mathcal{B}^{-} .
$$

$\mathcal{B}^{+}$and $\mathcal{B}^{-}$reduce to the form given in Eq. (86):

$$
A^{\mathcal{B}+}(\boldsymbol{k}, \boldsymbol{k}, \tau)=A^{\mathcal{B}-}(\boldsymbol{k}, \boldsymbol{k}, \tau)=\beta \frac{\lambda m H^{3}}{2 i f}\left(\begin{array}{cc}
\mathbf{0} & -\mathbf{I}_{2} \\
\mathbf{I}_{2} & \mathbf{0}
\end{array}\right) .
$$

From the axion field equation in the slow-roll regime, we have $\frac{\ddot{\varphi}}{H \dot{\varphi}} \ll 1$, and

$$
3 H \dot{\varphi}+V_{\varphi, \text { eff }} \simeq 0,
$$

where $V_{\varphi, \text { eff }}=V_{\varphi}+\frac{3 \lambda g_{A}}{f} \psi^{2}(\dot{\psi}+H \psi)$. The validity of the perturbation and slow-roll dynamics requires $\mathcal{B}$ to be at most of the order of the slow-roll suppressed terms, e.g.,

$$
\mathcal{B} \ll H \dot{\varphi} .
$$




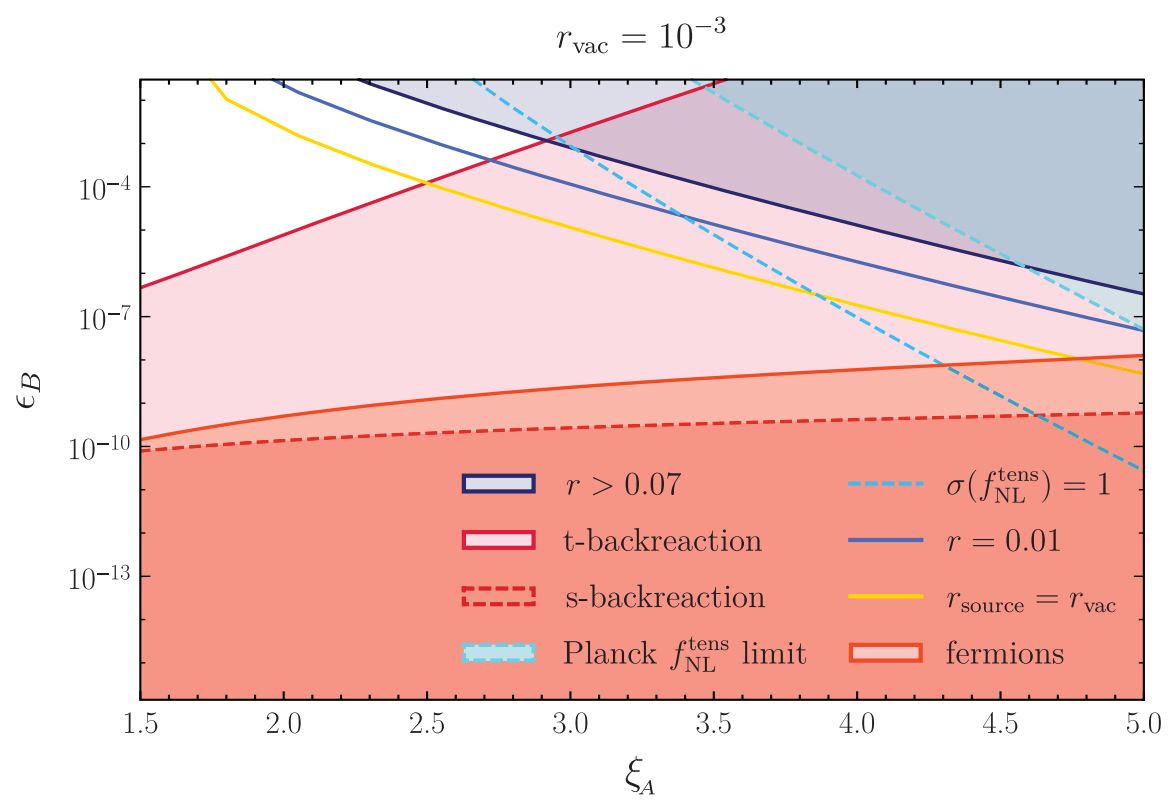

FIG. 2. We plot the energy density fraction of the gauge field $\epsilon_{B}$ as a function of the effective mass of the gauge field, $\xi_{A}$. Excluded parameter space with $r_{\text {vac }}=10^{-3}$. The blue shaded area is excluded by the tensor-to-scalar ratio, the light red area by the large tensor backreaction discussed in [33]. The cyan area by the tensor non-Gaussianity, and the dark red area by the Schwinger pair creation of scalar fields discussed in [31]. The blue and yellow lines show $r=10^{-2}$ and $r_{\text {source }}=r_{\text {vac }}$, respectively, while the dashed cyan line shows $f_{\mathrm{NL}}^{\text {tens }}=1$. The bound from the fermion particle production, depicted by the orange solid line (for $m=H$ and $\xi_{\varphi}=1$ ) and the area underneath, does not lead to additional bounds on the observationally relevant parameter space. The $\xi_{A}$ on the horizontal axis is the same as $m_{Q}$ in $[12,17,18]$.

Interestingly, however we find that our choice of initial conditions that makes the Hamiltonian diagonalized yields

$$
A_{\alpha \beta}^{\mathcal{B}^{s}}(\boldsymbol{k}, \boldsymbol{k}, \tau)\left(V_{s, \boldsymbol{k}, \alpha}^{*}(\tau) V_{s, \boldsymbol{k}, \beta}(\tau)-U_{s, \boldsymbol{k}, \beta}(\tau) U_{s, \boldsymbol{k}, \alpha}^{*}(\tau)\right)=0,
$$

which implies that

$$
\mathcal{B}=0 .
$$

Therefore, the particle production does not lead to any backreaction on the axion background. Note that this is the direct result of our quantization scheme based on diagonalization of the Hamiltonian. Setting the initial condition based on charge conjugation symmetry which does not diagonalize the Hamiltonian at asymptotic past, leads to a different and nonvanishing value for $\nabla_{\mu} J_{5}^{\mu}$, which is worked out in [47].

The computation so far has been done effectively at tree level. A one-loop effect, which has not been included consistently (see [38] for a related work with massless fermions), is the chiral anomaly, i.e., a quantum correction to the expectation value of $\nabla_{\mu} J^{\mu 5}$, equal to $g_{A}^{2} \operatorname{Tr}\left(\boldsymbol{F}_{\mu \nu} \tilde{\boldsymbol{F}}^{\mu \nu}\right) /\left(16 \pi^{2}\right)$ [44] which is $\approx 3 g_{A}^{3} \psi^{3} H /\left(4 \pi^{2}\right)$. Since $\mathcal{B}=(\beta \lambda) /(2 f) \nabla_{\mu} J^{\mu 5}$, using $g_{A} \lambda \partial_{\tau} \varphi /(a f) \approx$ $2 H\left(\xi_{A}+\xi_{A}^{-1}\right)$, Eq. (96) yields

$$
\left(\frac{f}{H}\right)^{2} \gg \frac{3}{16 \pi^{2}} \beta \xi_{A}^{2}\left(\xi_{A}^{2}+1\right)
$$

Since the right-hand side is always of the order of unity, the backreaction is never important when $f \gg H$.

\section{Parameter space of a model}

Our method applies to models in which inflation is driven by the axion-gauge field sector [1-4], as well as to those in which the axion and gauge fields are in a spectator sector [12]. For concreteness, we consider the latter model and compare our results with observational bounds on the following spectator model:

$$
\begin{aligned}
S= & S_{\mathrm{EH}}+S_{\phi}+S_{\text {spec }}+S_{\text {fermion }}+S_{\text {int }}, \\
S_{\text {spec }}= & \int \mathrm{d}^{4} x \sqrt{-g}\left[\frac{1}{2} \partial_{\mu} \varphi \partial^{\mu} \varphi-V(\varphi)\right. \\
& \left.-\frac{1}{2} \operatorname{Tr}\left(\boldsymbol{F}_{\mu \nu} \boldsymbol{F}^{\mu \nu}\right)-\frac{\lambda \varphi}{2 f} \operatorname{Tr}\left(\boldsymbol{F}_{\mu \nu} \tilde{F}^{\mu \nu}\right)\right],
\end{aligned}
$$

where $S_{\mathrm{EH}}$ and $S_{\phi}$ are the Einstein-Hilbert and the inflaton actions, respectively, responsible for inflation of the universe, and $S_{\text {spec }}$ is the action of the spectator sector. It contains the axion-gauge field sector, where $\varphi$ is the axion with a potential $V(\varphi)$ and a decay constant $f$, and

$$
\mathbf{F}_{\mu \nu}=\nabla_{\mu} \mathbf{A}_{\nu}-\nabla_{\nu} \mathbf{A}_{\mu}+i g_{A}\left(\mathbf{A}_{\mu} \mathbf{A}_{\nu}-\mathbf{A}_{\nu} \mathbf{A}_{\mu}\right)
$$




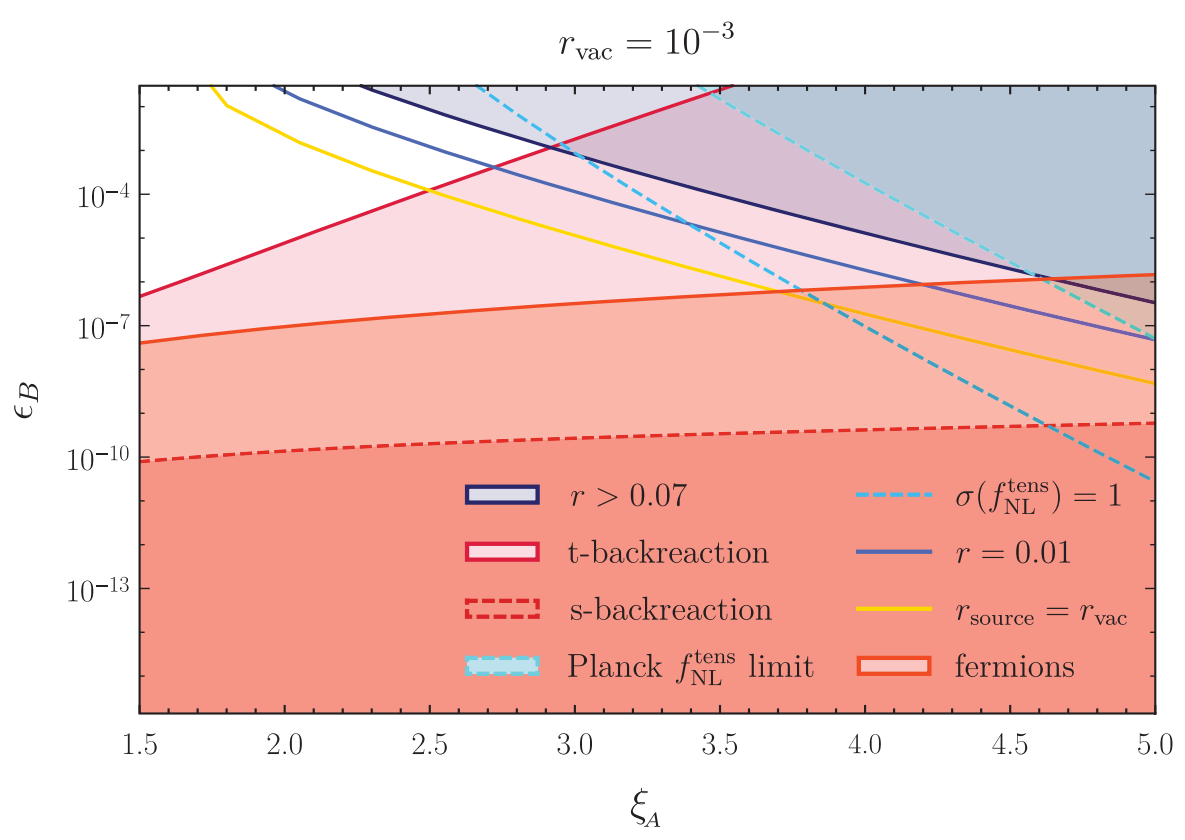

FIG. 3. Same as Fig. 2, but for $m=10 H$ and $\xi_{\varphi}=10$.

is the field strength tensor of the $S U(2)$ gauge fields. The last term in $S_{\text {spec }}$ is the Chern-Simons interaction, where $\lambda$ parametrizes its strength and $\tilde{\mathbf{F}}^{\mu \nu}$ is the dual of $\mathbf{F}_{\mu \nu}$.

For the bound on the backreaction of the gauge fields we use Eq. (92), which reduces to

$$
\epsilon_{B}<\xi_{A}^{3} \frac{10^{2} \pi^{2} A_{\mathrm{s}} r_{\mathrm{vac}}}{2} \frac{\mathcal{J}}{g_{A} H^{3}},
$$

where $\epsilon_{B} \equiv \xi_{A}^{4} H^{2} /\left(g_{A}^{2} m_{\mathrm{pl}}^{2}\right)$ is about 2 times the energy density fraction of the gauge field. We have also used the slow-roll relation $r_{\mathrm{vac}}=2 H^{2} /\left(A_{\mathrm{s}} \pi^{2} m_{\mathrm{pl}}^{2}\right)$ to parametrize the Hubble scale of inflation, i.e., $r_{\text {vac }}$ is the standard vacuum contribution to the tensor-to-scalar ratio in single-field slow-roll inflation. The amplitude of the curvature power spectrum is $A_{\mathrm{s}} \approx 2.2 \times 10^{-9}$ [48].

In Figs. 2-5, the orange solid line and the shaded area underneath it depict the inequality in Eq. (102), i.e., the regions where strong backreaction occurs due to the induced current. From these figures we can conclude that no additional constraint comes from the fermionic particle production.

Note that the contribution from chiral anomaly is small for the masses under consideration $\mu_{m} \gtrsim 1$, and also it is suppressed by a factor of $g_{A}^{2}$, (see footnote on page 3 for motivation behind this mass constraint, and see [38] for inclusion of one-loop effects for massless fermions).

\section{DISCUSSION}

We have studied the evolution of a Dirac field doublet, which is covariantly coupled to an axion and an isotropic $S U(2)$ gauge field background in de Sitter spacetime. We assumed the fermion field to have a Dirac mass term. Our work extends the previous work on fermion production from axion and Abelian $U(1)$ gauge fields [34-37], as well as on the simplest $S U(2)$ case with massless fermions [38].

We discovered that the $S U(2)$ background, in combination with the Dirac mass term, leads to nontrivial couplings between fermion components of different flavor and chirality. We then found a new convenient basis for the doublet of fermionic fields, given as a linear transformation in Fourier space of the original doublet, for which the action separates into two decoupled sectors. One of the subsystems is solvable analytically, whereas the other subsector is not and we solved it numerically.

Using these solutions, we computed the expectation values of the induced currents, which we used to estimate for what model parameters backreaction effects become important. More specifically, we considered the isotropic part of the $S U(2)$ matter current, as well as the 4-divergence of the axial current, which can be used to estimate the fermionic backreaction on the gauge field and axion backgrounds, respectively.

To find the vacuum expectation values of bilinearies in fermionic fields, such as the currents, we had to deal with UVdivergent integrals. To this end, we extended the idea of an existing instantaneous vacuum subtraction scheme [46], which involves the subtraction of the contribution of zeropoint fluctuations to the currents. We extended it to fermionic models with most generic Hamiltonians, which permit only a numerical treatment. We compared the results with independent regularization scheme, i.e the point-splitting method and found excellent agreement for $S_{+}$(the details of the latter method will appear in [47]). We find that the adiabatic vacuum subtraction scheme could not be utilized here, since 


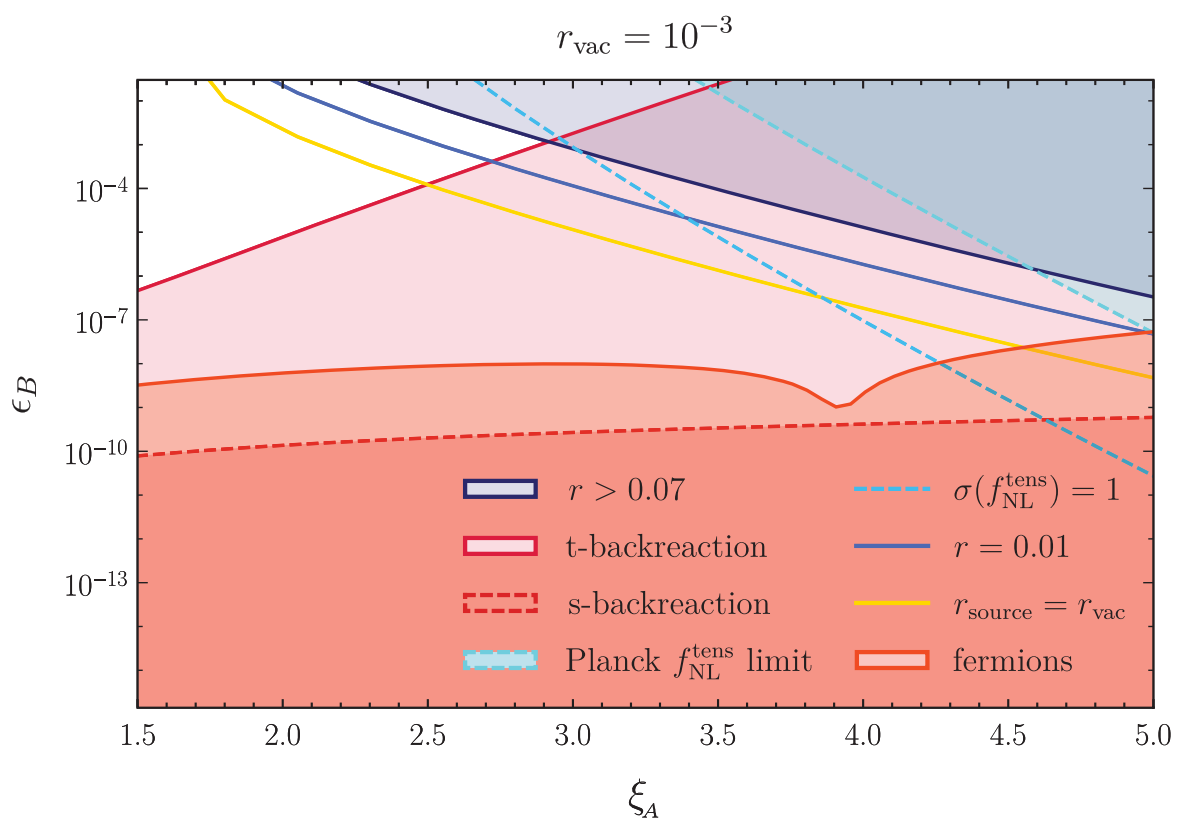

FIG. 4. Same as Fig. 2, but for $m=10 H$ and $\xi_{\varphi}=1$.

there are instants where adiabatic modes are ill behaved. We also made a careful distinction between the contributions of particles and antiparticles to the vacuum expectation values, which played an important role in the computation of the treelevel backreaction on the axion.

We showed that the $S U(2)$-background experiences strong backreaction due to fermions only for model parameters which are already excluded on observational and/or theoretical grounds (see Figs. 2-5), similarly to the case of scalars [31]. The tree-level expectation value of the 4-divergence of the axial current vanishes. We then estimated when the chiral anomaly, which is a loop effect, becomes important for the backreaction on the axion background. We find that backreaction remains unimportant provided that $f \gg H$.

We conclude that the background dynamics of an axion$S U$ (2) gauge field spectator sector remains unaffected by production of fermions.

\section{ACKNOWLEDGMENTS}

We thank Peter Adshead, Giovanni Cabass, Marco Peloso, Lorenzo Sorbo, and Yuki Watanabe for useful discussions. We are especially grateful to Eiichiro Komatsu for insightful discussions and comments on the manuscript.

\section{APPENDIX A: MATHEMATICAL TOOLS}

In this Appendix, we review some mathematical tools that we use throughout this work. These include the definition of the direct sum, Kronecker product, the spin connection, and some asymptotic forms of Whittaker functions.

The vector space, $\mathbf{V}$, is the direct sum of two subspaces, $\mathbf{U}_{1}$ and $\mathbf{U}_{2}$, as

$$
\mathbf{V}=\mathbf{U}_{1} \oplus \mathbf{U}_{2},
$$

if and only if $\mathbf{V}=\mathbf{U}_{1}+\mathbf{U}_{2}$, and $\mathbf{U}_{1}$ and $\mathbf{U}_{2}$ are independent.

The Kronecker product of two matrices, $\mathbf{A}_{m \times n}$ and $\mathbf{B}_{q \times p}$, is defined as a $m p \times n q$ block matrix given by

$$
\mathbf{A} \otimes \mathbf{B}=\left(\begin{array}{ccc}
A_{11} \mathbf{B} & \ldots & A_{1 n} \mathbf{B} \\
& \ddots & \\
A_{m 1} \mathbf{B} & \ldots & A_{m n} \mathbf{B}
\end{array}\right)
$$

The 8 -spinor covariant derivative in (7) is

$$
D_{\mu} \otimes \gamma^{\alpha} \tilde{\Psi} \equiv\left(\mathbf{I}_{2} \nabla_{\mu}-i g_{A} \mathbf{A}_{\mu}\right) \otimes \gamma^{\alpha} \tilde{\Psi},
$$

where the spin covariant derivative is

$$
\nabla_{\mu} \tilde{\Psi}=\left[\mathrm{I}_{4} \partial_{\mu}+\omega_{\mu}\right] \tilde{\Psi}
$$

with $\omega_{\mu}$ being the spin connections,

$$
\omega_{\mu}=-\frac{i}{2} \omega_{\mu}^{\alpha \beta} \sigma_{\alpha \beta},
$$

and $\sigma_{\alpha \beta}=\frac{i}{4}\left[\gamma_{\alpha}, \gamma_{\beta}\right]$ being the spinor generators of the Lorentz algebra. The elements of the spin connection $\omega_{\mu}^{\alpha \beta}$ are given by

$$
\omega_{\mu}{ }^{\alpha \beta}=\mathbf{e}_{\nu}{ }^{\alpha} \nabla_{\mu} \mathbf{e}^{\nu \beta} .
$$

In FLRW spacetime using the conformal time, the vierbeins are 


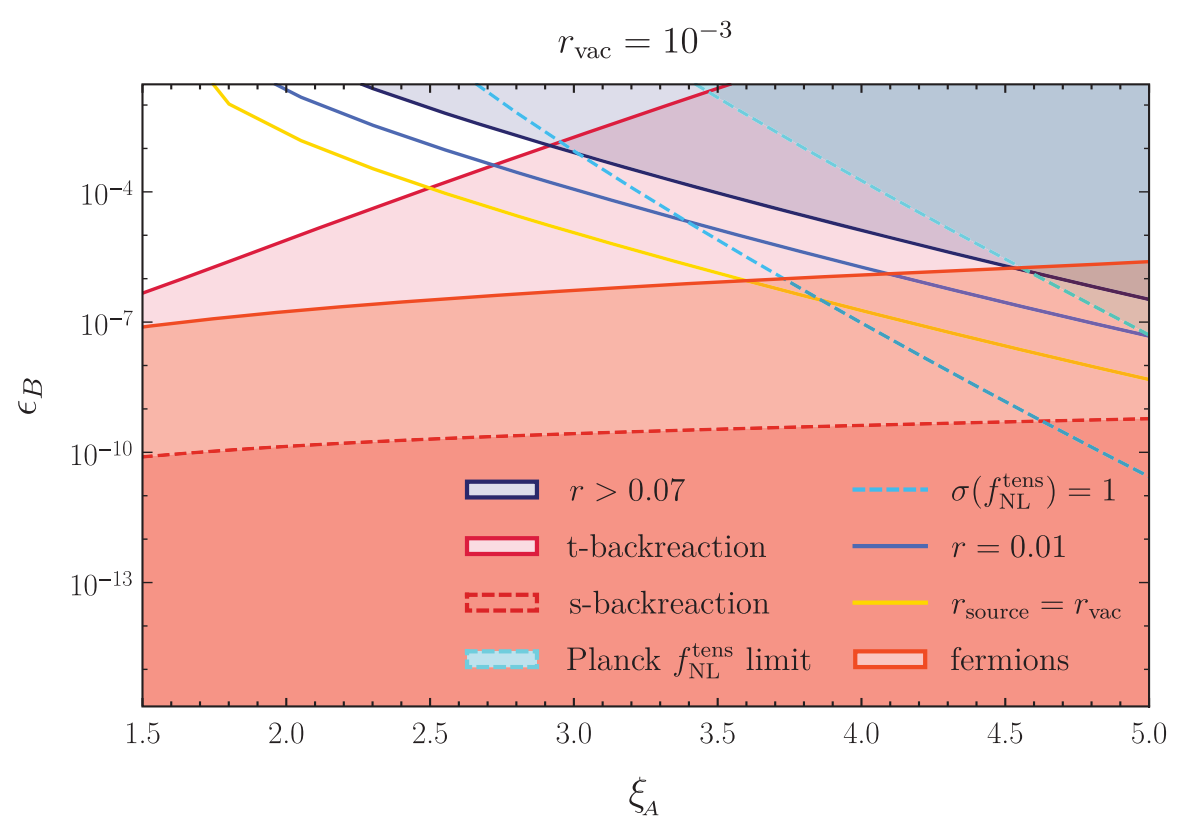

FIG. 5. Same as Fig. 2, but for $m=H$ and $\xi_{\varphi}=10$.

$$
\mathbf{e}^{\mu}{ }_{\alpha}=a(\tau)^{-1} \delta_{\alpha}^{\mu},
$$

and the only nonzero components of the spin connection coefficients are

$$
\omega_{\mu}{ }^{i 0}=-\omega_{\mu}{ }^{0 i}=-\mathcal{H} \delta_{\mu}^{i} .
$$

Whittaker functions $W_{\kappa, \mu}(z)$ and $M_{\kappa, \mu}(z)$ take the following asymptotic forms in the limit $|z| \rightarrow \infty$ :

$$
\begin{aligned}
W_{\kappa, \mu}(z) \rightarrow & z^{\kappa} e^{-z / 2}, \\
M_{\kappa, \mu}(z) \rightarrow & \Gamma(2 \mu+1)\left(\frac{i(-1)^{\mu-\kappa} z^{\kappa} e^{-z / 2}}{\Gamma\left(-\kappa+\mu+\frac{1}{2}\right)}\right. \\
& \left.+\frac{z^{-\kappa} e^{z / 2}}{\Gamma\left(-\kappa+\mu+\frac{1}{2}\right)}\right)
\end{aligned}
$$

where $|\arg z|<\frac{3}{2} \pi$. Thus, for a complex $\kappa$, we have

$$
\lim _{\tau \rightarrow-\infty} \frac{(2 \tilde{\tau})^{-\kappa_{\mathrm{R}}}}{\sqrt{2 k}} e^{-\kappa_{\mathrm{I}} \pi / 2} W_{\kappa, \mu}(-2 i \tilde{\tau})=\frac{1}{\sqrt{2 k}} e^{-i k \tau},
$$

where $\kappa_{\mathrm{R}}$ and $\kappa_{\mathrm{I}}$ are the real and imaginary parts of $\kappa$. Therefore,

$$
\frac{(2 \tilde{\tau})^{-\kappa_{\mathrm{R}}}}{(2 \pi)^{3 / 2} \sqrt{2 k}} e^{-\kappa_{\mathrm{I}} \pi / 2} W_{\kappa, \mu}(-2 i \tilde{\tau})
$$

represents the positive frequency solutions in the asymptotic past, i.e., the Bunch-Davies vacuum.

\section{APPENDIX B: THE SPINOR SUBSPACES, $\tilde{\psi}=\psi^{+} \oplus \psi^{-}$}

In this Appendix, our aim is to reduce an 8 -spinor into two 4-spinors. Again we recall that the tilde denotes $8 \times 8$ matrices, $4 \times 4$ matrices remained unaltered; and the $2 \times 2$ matrices are presented in boldface. To find the spinor subspaces, it is convenient to apply the following chain of unitary transformations: First, in Sec. B 1 we review the Weyl representation, second, in. Section B 2 we define the new extended helicity basis to transform $\psi_{L}$ and $\psi_{R}$ to $\psi^{+}$and $\psi^{-}$, and in Sec. B 3 we transform each of the subspinors to the Dirac representation:

$$
\tilde{\psi}: \underbrace{\left(\begin{array}{c}
\psi^{1} \\
\psi^{2}
\end{array}\right)}_{\text {flavor }} \stackrel{\tilde{T}_{1}}{\rightarrow} \underbrace{\left(\begin{array}{c}
\psi_{L} \\
\psi_{R}
\end{array}\right)}_{\text {chiral }} \stackrel{\tilde{T}_{2}}{\rightarrow} \underbrace{\left(\begin{array}{c}
\psi^{+} \\
\psi^{-}
\end{array}\right)}_{\text {extended helicity }} .
$$

Note that the matrix $\tilde{P}_{ \pm}(\boldsymbol{k})$ in Eq. (18) consists of all the above chain of unitary transformations.

\section{Weyl representation}

The 8-spinor can be decomposed into two chirality states by the projection operators

$$
\tilde{P}_{L, R}=\mathbf{I}_{2} \otimes\left(\frac{\mathrm{I}_{4} \mp \gamma^{5}}{2}\right)
$$

such that left- and right-handed components are given by

$$
\tilde{\psi}_{L, R}=\tilde{P}_{L, R} \tilde{\psi} .
$$


In (B2) the minus and plus signs are for $\mathrm{L}$ and $\mathrm{R}$, respectively.

The spinor in the flavor (Dirac) frame transforms into the chiral frame as

$$
\tilde{T}_{1}\left(\begin{array}{c}
\psi^{1} \\
\psi^{2}
\end{array}\right)=\left(\begin{array}{c}
\psi_{L} \\
\psi_{R}
\end{array}\right),
$$

where $\tilde{T}_{1}$ is the following unitary matrix:

$$
\tilde{T}_{1}=\frac{1}{\sqrt{2}}\left(\begin{array}{cccc}
\mathbf{I}_{2} & -\mathbf{I}_{2} & \mathbf{0} & \mathbf{0} \\
\mathbf{0} & \mathbf{0} & \mathbf{I}_{2} & -\mathbf{I}_{2} \\
\mathbf{I}_{2} & \mathbf{I}_{2} & \mathbf{0} & \mathbf{0} \\
\mathbf{0} & \mathbf{0} & \mathbf{I}_{2} & \mathbf{I}_{2}
\end{array}\right) .
$$

Moreover, the $\mathbf{I}_{2} \otimes \gamma^{\alpha}$ operators transform as

$$
\tilde{T}_{1}\left(\mathbf{I}_{2} \otimes \gamma^{\alpha}\right) \tilde{T}_{1}^{-1}=\left(\begin{array}{cccc}
\mathbf{0} & \mathbf{0} & \boldsymbol{\sigma}^{\alpha} & \mathbf{0} \\
\mathbf{0} & \mathbf{0} & \mathbf{0} & \boldsymbol{\sigma}^{\alpha} \\
\overline{\boldsymbol{\sigma}}^{\alpha} & \mathbf{0} & \mathbf{0} & \mathbf{0} \\
\mathbf{0} & \overline{\boldsymbol{\sigma}}^{\alpha} & \mathbf{0} & \mathbf{0}
\end{array}\right),
$$

where

$$
\boldsymbol{\sigma}^{\alpha}=\left(\mathbf{I}_{2}, \boldsymbol{\sigma}^{i}\right) \quad \text { and } \quad \overline{\boldsymbol{\sigma}}^{\alpha}=\left(\mathbf{I}_{2},-\boldsymbol{\sigma}^{i}\right) .
$$

The (flat space) gamma matrices in the Weyl representation are

$$
\gamma_{\mathrm{W}}^{\alpha}=\left(\begin{array}{cc}
\mathbf{0} & \boldsymbol{\sigma}^{\alpha} \\
\overline{\boldsymbol{\sigma}}^{\alpha} & \mathbf{0}
\end{array}\right) \text { and } \gamma_{\mathrm{W}}^{5}=\left(\begin{array}{cc}
-\mathbf{I}_{2} & \mathbf{0} \\
\mathbf{0} & \mathbf{I}_{2}
\end{array}\right)
$$

In this frame, the action in (13) is given as

$$
S=\int \mathrm{d} \tau \mathrm{d} k^{3}\left(\bar{\psi}_{R, \mathbf{k}} \bar{\psi}_{L, \mathbf{k}}\right) \tilde{\mathrm{L}}_{\mathbf{k}}(\tau)\left(\begin{array}{c}
\psi_{L, \mathbf{k}} \\
\psi_{R, \mathbf{k}}
\end{array}\right),
$$

where $\tilde{L}_{\mathbf{k}}(\tau)$ is the following $8 \times 8$ operator:

$\tilde{\mathrm{L}}_{\mathbf{k}}(\tau) \equiv i\left(\begin{array}{cc}i \mu_{\mathrm{m}} \mathcal{H I}_{4} & \mathrm{I}_{4} \partial_{\tau}+i \Sigma_{4}(\tau, \boldsymbol{k}) \\ \mathrm{I}_{4} \partial_{\tau}-i \Sigma_{4}(\tau, \boldsymbol{k}) & i \mu_{\mathrm{m}} \mathcal{H} \mathrm{I}_{4}\end{array}\right)$,

where $\Sigma_{4}$ is the following $4 \times 4$ operator:

$$
\Sigma_{4}(\tau, \boldsymbol{k})=\mathbf{I}_{2} \otimes k^{i} \cdot \boldsymbol{\sigma}^{i}+\mathcal{H}\left(2 \xi_{\varphi} \mathrm{I}_{4}-\frac{\xi_{A}}{2} \boldsymbol{\sigma}^{i} \otimes \boldsymbol{\sigma}^{j} \delta_{i j}\right) .
$$

In the absence of the mass term, the system in (B8) is decomposed into two independent subsectors in terms of the left- and right-handed fields. However, in the massive case, we need to take one step further and expand the fields in the extended helicity states.

\section{Extended helicity states}

The aim here is to write the $4 \times 4$ operator, $\Sigma_{4}$, in (B9) as a block diagonal matrix. For that we need to find the common eigenstates of the $4 \times 4$ helicity operator, $\mathbf{I}_{2} \otimes k^{i} \cdot \boldsymbol{\sigma}^{i}$, and $\boldsymbol{\sigma}^{i} \otimes \boldsymbol{\sigma}_{i}$. These two have only two common eigenstates and therefore, it is not possible to fully diagonalize $\Sigma_{4}$. Nevertheless, it can be block diagonalized and decomposed into two subspaces.

For a given momentum, $k^{\alpha}$, the orthonormal eigenstates for the helicity operator, $\mathbf{I}_{2} \otimes k^{i} \cdot \boldsymbol{\sigma}^{i}$, are

$$
e_{+}^{+}\left(k^{\alpha}\right)=\frac{\check{k}^{\alpha} \overline{\boldsymbol{\sigma}}_{\alpha} \otimes \check{k}^{\beta} \overline{\boldsymbol{\sigma}}_{\beta}}{2 k\left(k+k^{3}\right)}\left(\begin{array}{l}
1 \\
0 \\
0 \\
0
\end{array}\right),
$$

$$
e_{-}^{+}\left(k^{\alpha}\right)=-\frac{\check{k}^{\alpha} \boldsymbol{\sigma}_{\alpha} \otimes \check{k}^{\beta} \boldsymbol{\sigma}_{\beta}}{2 k\left(k+k^{3}\right)}\left(\begin{array}{l}
0 \\
0 \\
0 \\
1
\end{array}\right) \text {, }
$$

$$
e_{+}^{-}\left(k^{\alpha}\right)=-\frac{\check{k}^{\alpha} \boldsymbol{\sigma}_{\alpha} \otimes \check{k}^{\beta} \overline{\boldsymbol{\sigma}}_{\beta}}{2 k\left(k+k^{3}\right)}\left(\begin{array}{l}
0 \\
0 \\
1 \\
0
\end{array}\right) \text {, }
$$

$$
e_{-}^{-}\left(k^{\alpha}\right)=-\frac{\check{k}^{\alpha} \overline{\boldsymbol{\sigma}}_{\alpha} \otimes \check{k}^{\beta} \boldsymbol{\sigma}_{\beta}}{2 k\left(k+k^{3}\right)}\left(\begin{array}{l}
0 \\
1 \\
0 \\
0
\end{array}\right) \text {, }
$$

where $\boldsymbol{\sigma}^{\alpha}$ and $\overline{\boldsymbol{\sigma}}^{\alpha}$ are given in (B6), and their indices are lowered with the Minkowski metric, i.e., $\boldsymbol{\sigma}_{\alpha}=\eta_{\alpha \beta} \boldsymbol{\sigma}^{\beta}$, and $\breve{k}^{\alpha}$ is a four vector given as

$$
\check{k}^{\alpha} \equiv(k, \boldsymbol{k}),
$$

where $k=\sqrt{k^{i} \cdot k^{i}}$. Notice that $\breve{k}^{\alpha}$ is the four momentum of the massless field, but for the massive cases it is just a mathematical tool.

The $e^{p}{ }_{s}\left(k^{\alpha}\right)$ with $p= \pm 1$ and $s= \pm 1$ satisfies the eigenstate equation

$$
\mathbf{I}_{2} \otimes k^{i} \cdot \boldsymbol{\sigma}^{i} e^{p}{ }_{s}\left(k^{\alpha}\right)=s k e^{p}{ }_{s}\left(k^{\alpha}\right),
$$

and the orthonormality condition

$$
e_{s}^{p^{\dagger}}\left(k^{\alpha}\right) \cdot e^{p^{\prime}{ }_{s^{\prime}}}\left(k^{\alpha}\right)=\delta_{s s^{\prime}} \delta^{p p^{\prime}} .
$$

The $p=+1$ objects are also eigenvectors of $\Sigma_{4}(\tau, \boldsymbol{k})$ in (B10):

$$
\Sigma_{4}(\tau, \boldsymbol{k}) e_{s}^{+}\left(k^{\alpha}\right)=\lambda_{s}^{+} e_{s}^{+}\left(k^{\alpha}\right)
$$


where $\lambda_{s}^{+}=s k+\left(-\frac{1}{2} \xi_{A}+2 \xi_{\varphi}\right) \mathcal{H}$.

Since $e^{p}{ }_{s}\left(k^{\alpha}\right)$ make an orthonormal basis, we can expand fields and matrices in that frame by using the unitary transformation

$$
\begin{aligned}
R_{\mathbf{k}} & =\left[e_{+}^{+}\left(k^{\alpha}\right) e_{-}^{+}\left(k^{\alpha}\right) e_{-}^{-}\left(k^{\alpha}\right) e_{+}^{-}\left(k^{\alpha}\right)\right] \\
& =\left(\begin{array}{llll}
e_{+1}^{+} & e_{-1}^{+} & e_{-1}^{-} & e_{+1}^{-} \\
e_{+2}^{+} & e_{-2}^{+} & e_{-2}^{-} & e_{+2}^{-} \\
e_{+3}^{+} & e_{-3}^{+} & e_{-3}^{-} & e_{+3}^{-} \\
e_{+4}^{+} & e_{-4}^{+} & e_{-4}^{-} & e_{+4}^{-}
\end{array}\right)
\end{aligned}
$$

where $e^{p}{ }_{s i}$ is the $i$ th element of the $e^{p}{ }_{s}\left(k^{\alpha}\right)$.

For each given momentum, $\boldsymbol{k}, R_{\mathbf{k}}$ takes the Weyl spinors to their helicity frame. The normal spinor basis in this frame are

$$
\begin{array}{ll}
e_{L+}=\left(\begin{array}{c}
\mathbf{E}_{+} \\
\mathbf{0}
\end{array}\right), & e_{L-}=\left(\begin{array}{c}
\mathbf{E}_{-} \\
\mathbf{0}
\end{array}\right) \\
e_{R+}=\left(\begin{array}{c}
\mathbf{0} \\
\mathbf{E}_{+}
\end{array}\right), & e_{R-}=\left(\begin{array}{c}
\mathbf{0} \\
\mathbf{E}_{-}
\end{array}\right),
\end{array}
$$

where $\mathbf{E}_{+}$and $\mathbf{E}_{-}$are the following 2-spinor basis:

$$
\mathbf{E}_{+}=\left(\begin{array}{l}
1 \\
0
\end{array}\right) \quad \text { and } \quad \mathbf{E}_{-}=\left(\begin{array}{l}
0 \\
1
\end{array}\right)
$$

In the frame of helicity, $\Sigma_{4}(\tau, \boldsymbol{k})$ takes a block diagonal form

$$
\check{\Sigma}_{4}(\tau, k)=R_{\mathbf{k}}^{-1} \Sigma_{4}(\tau, \boldsymbol{k}) R_{\mathbf{k}}=\left(\begin{array}{cc}
\check{\boldsymbol{\Sigma}}^{+} & 0 \\
0 & \check{\boldsymbol{\Sigma}}^{-}
\end{array}\right),
$$

where $\check{\boldsymbol{\Sigma}}^{ \pm}$are $2 \times 2$ matrices defined as

$$
\begin{aligned}
& \check{\boldsymbol{\Sigma}}^{+} \equiv k \boldsymbol{\sigma}^{3}+\left(2 \xi_{\varphi}-\frac{\xi_{A}}{2}\right) \mathcal{H} \mathbf{I}_{2}, \\
& \check{\boldsymbol{\Sigma}}^{-} \equiv-k \boldsymbol{\sigma}^{3}+\left(2 \xi_{\varphi}+\frac{\xi_{A}}{2}\right) \mathcal{H} \mathbf{I}_{2}-\xi_{A} \mathcal{H} \boldsymbol{\sigma}^{1} .
\end{aligned}
$$

The 8-spinor in the Weyl representation can be written in terms of the helicity states as

$$
\tilde{R}_{\mathbf{k}}\left(\begin{array}{c}
\psi_{L, \mathbf{k}} \\
\psi_{R, \mathbf{k}}
\end{array}\right)=\left(\begin{array}{c}
\boldsymbol{\Psi}_{L, \mathbf{k}}^{+} \\
\boldsymbol{\Psi}_{L, \mathbf{k}}^{-} \\
\boldsymbol{\Psi}_{R, \mathbf{k}}^{+} \\
\boldsymbol{\Psi}_{R, \mathbf{k}}^{-}
\end{array}\right),
$$

where $\tilde{R}_{\mathbf{k}}$ is the following $8 \times 8$ unitary operator:

$$
\tilde{R}_{\mathbf{k}} \equiv \mathbf{I}_{2} \otimes R_{\mathbf{k}}^{-1}
$$

The Lagrangian operator in (B9) is also transformed as

$$
\begin{aligned}
\check{L}_{\mathbf{k}}(\tau) & =\tilde{R}_{\mathbf{k}} \tilde{L}_{\mathbf{k}}(\tau) \tilde{R}_{\mathbf{k}}^{-1} \\
& =i\left(\begin{array}{cc}
i \mu_{\mathrm{m}} \mathcal{H} \mathrm{I}_{4} & \mathrm{I}_{4} \partial_{\tau}+i \check{\Sigma}_{4}(\tau, \boldsymbol{k}) \\
\mathrm{I}_{4} \partial_{\tau}-i \check{\Sigma}_{4}(\tau, \boldsymbol{k}) & i \mu_{\mathrm{m}} \mathcal{H I}_{4}
\end{array}\right),
\end{aligned}
$$

which decouples the plus and minus spinors in (B21). This split would be clearer if we take another unitary transformation,

$$
\tilde{S}=\left(\begin{array}{cccc}
\mathbf{I}_{2} & 0 & 0 & 0 \\
0 & 0 & \mathbf{I}_{2} & 0 \\
0 & \mathbf{I}_{2} & 0 & 0 \\
0 & 0 & 0 & \mathbf{I}_{2}
\end{array}\right),
$$

and define $\tilde{T}_{2, \mathbf{k}}$ as

$$
\tilde{T}_{2, \mathbf{k}} \equiv \tilde{S} \tilde{R}_{\mathbf{k}} \cdot
$$

Under the action of $\tilde{T}_{2, \mathbf{k}}$, the 8 -spinor transforms as

$$
\tilde{T}_{2, \mathbf{k}}\left(\begin{array}{c}
\psi_{L, \mathbf{k}} \\
\psi_{R, \mathbf{k}}
\end{array}\right)=\left(\begin{array}{c}
\psi_{\mathbf{k}}^{+} \\
\psi_{\mathbf{k}}^{-}
\end{array}\right),
$$

and the Lagrangian operator becomes the following block diagonal $8 \times 8$ matrix:

$$
L_{\mathbf{k}}(\tau)=\tilde{T}_{2, \mathbf{k}} \tilde{L}_{\mathbf{k}}(\tau) \tilde{T}_{2, \mathbf{k}}^{-1}=\left(\begin{array}{cc}
L_{\mathbf{k}}^{+}(\tau) & 0 \\
0 & L_{\mathbf{k}}^{-}(\tau)
\end{array}\right),
$$

where $L_{\mathbf{k}}^{ \pm}(\tau)$ are the following $4 \times 4$ operations

$L_{\mathbf{k}}^{ \pm}(\tau) \equiv i\left(\begin{array}{cc}i \mu_{\mathrm{m}} \mathcal{H} \mathbf{I}_{2} & \mathbf{I}_{2} \partial_{\tau}+i \check{\boldsymbol{\Sigma}}^{ \pm}(\tau, \mathbf{k}) \\ \mathbf{I}_{2} \partial_{\tau}-i \check{\boldsymbol{\Sigma}}^{ \pm}(\tau, \mathbf{k}) & i \mu_{\mathrm{m}} \mathcal{H} \mathbf{I}_{2}\end{array}\right)$.

Here, $\check{\boldsymbol{\Sigma}}^{ \pm}(\tau, \mathbf{k})$ are given in (B20). Therefore, the theory in (B8) splits into two subsectors in terms of the plus and minus spinors as

$$
S[\tilde{\psi}]=S_{+}\left[\tilde{\psi}^{+}\right]+S_{-}\left[\tilde{\psi}^{-}\right],
$$

where

$$
S_{ \pm}=\int \frac{\mathrm{d} \tau \mathrm{d} k^{3}}{(2 \pi)^{3}} \bar{\psi}_{\mathbf{k}}^{ \pm} \tilde{\mathrm{L}}_{\mathbf{k}}^{ \pm}(\tau) \psi_{\mathbf{k}}^{ \pm}
$$

The $\tilde{\mathrm{L}}_{\mathbf{k}}^{ \pm}(\tau)$ operators are given as

$$
\begin{aligned}
\tilde{\mathrm{L}}_{\mathbf{k}}^{+}(\tau) \equiv & {\left[i \gamma_{\mathrm{W}}^{0} \partial_{\tau}-k \gamma_{\mathrm{W}}^{3}-\left(2 \xi_{\varphi}-\frac{\xi_{A}}{2}\right) \mathcal{H} \lambda_{4}-\mu_{\mathrm{m}} \mathcal{H} \mathrm{I}_{4}\right], } \\
\tilde{\mathrm{L}}_{\mathbf{k}}^{-}(\tau) \equiv & {\left[i \gamma_{\mathrm{W}}^{0} \partial_{\tau}+k \gamma_{\mathrm{W}}^{3}+\gamma_{\mathrm{W}}^{1} \xi_{A} \mathcal{H}-\left(2 \xi_{\varphi}+\frac{\xi_{A}}{2}\right) \mathcal{H} \lambda_{4}\right.} \\
& \left.-\mu_{\mathrm{m}} \mathcal{H} \mathrm{I}_{4}\right],
\end{aligned}
$$


where $\gamma_{\mathrm{W}}^{\alpha} \mathrm{s}$ are the gamma matrices in the Weyl representation and $\lambda_{\mathrm{W}}$ is

$$
\lambda_{4} \equiv\left(\begin{array}{cc}
0 & \mathbf{I}_{2} \\
-\mathbf{I}_{2} & 0
\end{array}\right)
$$

We thus showed that our 8-spinor space splits into two irreducible representations,

$$
\tilde{\psi}=\psi^{+} \oplus \psi^{-},
$$

in terms of two 4-spinors $\psi^{+}$and $\psi^{-}$.

\section{Subspinors in Dirac frame}

Up to this point, we have split the spinor space into two subspaces but each of the supspinors are still in their Weyl representation [see (B26)]

$$
\tilde{V}_{\mathbf{k}}\left(\begin{array}{c}
\psi_{L, \mathbf{k}} \\
\psi_{R, \mathbf{k}}
\end{array}\right)=\left(\begin{array}{c}
\boldsymbol{\Psi}_{L, \mathbf{k}}^{+} \\
\boldsymbol{\Psi}_{R, \mathbf{k}}^{+} \\
\boldsymbol{\Psi}_{L, \mathbf{k}}^{-} \\
\boldsymbol{\Psi}_{R, \mathbf{k}}^{-}
\end{array}\right) .
$$

The 4-spinors can be transformed to their Dirac representation as

$$
\psi_{\mathrm{D}}^{ \pm}=\mathcal{D} \psi^{ \pm}
$$

where

$$
\mathcal{D}=\frac{1}{\sqrt{2}}\left(\begin{array}{cc}
\mathbf{I}_{2} & \mathbf{I}_{2} \\
-\mathbf{I}_{2} & \mathbf{I}_{2}
\end{array}\right) .
$$

The gamma matrices in the Weyl representation, $\gamma_{\mathrm{W}}^{\alpha}$, and Dirac representation, $\gamma^{\alpha}$, are related as $\gamma^{\alpha}=\mathcal{D} \gamma_{\mathrm{W}}^{\alpha} \mathcal{D}^{-1}$. Moreover, the Lagrangian operators of each of the subspaces in (B31) and (B32) take the following forms in the Dirac representation:

$$
\begin{aligned}
\tilde{\mathrm{L}}_{\mathbf{k}, \mathrm{D}}^{+}(\tau) \equiv & {\left[i \gamma^{0} \partial_{\tau}-k \gamma^{3}-\left(2 \xi_{\varphi}-\frac{\xi_{A}}{2}\right) \mathcal{H} \lambda_{4}-\mu_{\mathrm{m}} \mathcal{H} \mathrm{I}_{4}\right] } \\
\tilde{\mathrm{L}}_{\mathbf{k}, \mathrm{D}}^{-}(\tau) \equiv & {\left[i \gamma^{0} \partial_{\tau}+k \gamma^{3}+\gamma^{1} \xi_{A} \mathcal{H}-\left(2 \xi_{\varphi}+\frac{\xi_{A}}{2}\right) \mathcal{H} \lambda_{4}\right.} \\
& \left.-\mu_{\mathrm{m}} \mathcal{H} \mathrm{I}_{4}\right] .
\end{aligned}
$$

In the Dirac-helicity frame which we introduced above, the Dirac fields can be expanded as

$$
\psi_{\mathbf{k}}^{ \pm}=\sum_{s= \pm}\left(\begin{array}{c}
\psi_{s}^{ \pm \uparrow}(\tau, k) \mathbf{E}_{s} \\
s \psi_{s}^{ \pm \downarrow}(\tau, k) \mathbf{E}_{s}
\end{array}\right),
$$

where $\psi_{s}^{ \pm \uparrow}(\tau, k)$ and $\psi_{s}^{ \pm \downarrow}(\tau, k)$ are mode functions and $\mathbf{E}_{s}$ with $s= \pm 1$ are the two-spinor polarization states given in (B18).
Notice that $\gamma^{\alpha}$ are the gamma functions in the Dirac representation and $\lambda_{4}$ is the same in both Weyl and Dirac representations. For the sake of simplicity, in the main text we remove the $D$ subscript and write the fields and operators in the Dirac representation.

\section{APPENDIX C: HAMILTONIAN}

In this Appendix we derive and diagonalize the Hamiltonian of our model. The Hamiltonian is derived from the actions, given in Eqs. (21) and (22), by defining the Lagrangian $S_{ \pm} \equiv \int \mathrm{d} \tau L_{ \pm}$and then carrying out a Legendre transformation. We derive the Hamiltonians for $S_{+}$and $S_{-}$separately and diagonalize them afterwards.

Before we calculate the Hamiltonian, we need to explain the quantization procedure for the fermions in $S_{+}$and $S_{-}$.

\section{Quantization of the $S_{+}$fermions}

The quantization procedure for the $\psi_{k}^{+}$modes is the following. We first define the canonical conjugate momenta,

$$
\pi_{\boldsymbol{k}, \alpha}^{\psi+}=\frac{\delta S_{+}}{\delta \partial_{\tau} \psi_{k, \alpha}^{+}}=i \psi_{+, k, \alpha}^{*}
$$

where $\alpha$ runs from 1 to 4 . We then promote $\psi_{k}^{+}$and $\pi_{k}^{\psi+}$ to quantum operators, obeying the canonical equal-time anticommutation relations,

$$
\begin{aligned}
& \left\{\psi_{\boldsymbol{k}, \alpha}^{+}(\tau), \psi_{\boldsymbol{k}^{\prime}, \beta}^{+}(\tau)\right\}=0, \\
& \left\{\pi_{\boldsymbol{k}, \alpha}^{\psi+}(\tau), \pi_{\boldsymbol{k}^{\prime}, \beta}^{\psi+}(\tau)\right\}=0, \\
& \left\{\psi_{\boldsymbol{k}, \alpha}^{+}(\tau), \pi_{\boldsymbol{k}^{\prime}, \beta}^{\psi+}(\tau)\right\}=i(2 \pi)^{-3} \delta_{\alpha \beta} \delta^{(3)}\left(\boldsymbol{k}-\boldsymbol{k}^{\prime}\right) .
\end{aligned}
$$

We also impose that the time-independent coefficients in Eq. (28) are the standard anticommuting creation and annihilation operators, i.e.,

$$
\begin{aligned}
& \left\{a_{s, \boldsymbol{k}}^{+}, a_{s^{\prime}, \boldsymbol{k}^{\prime}}^{+\dagger}=\delta_{s s^{\prime}} \delta^{(3)}\left(\boldsymbol{k}-\boldsymbol{k}^{\prime}\right),\right. \\
& \left\{b_{s, \boldsymbol{k}}^{+}, b_{s^{\prime}, \boldsymbol{k}^{\prime}}^{+}\right\}=\delta_{s s^{\prime}} \delta^{(3)}\left(\boldsymbol{k}-\boldsymbol{k}^{\prime}\right),
\end{aligned}
$$

with all other anticommutators vanishing. The canonical quantization expressions in Eqs. (C2) and (C3) yield the following normalization condition:

$$
\begin{aligned}
& \sum_{s= \pm}\left[\left(U_{s, k}^{+}(\tau)\right)_{\alpha}\left(U_{s, k}^{+\dagger}(\tau)\right)_{\beta}+\left(V_{s, k}^{+}(\tau)\right)_{\alpha}\left(V_{s, k}^{+\dagger}(\tau)\right)_{\beta}\right] \\
& \quad=\delta_{\alpha \beta}(2 \pi)^{-3} .
\end{aligned}
$$

One can check that each term in the square brackets is indeed a constant, i.e., preserved by the equation of motion given in Eq. (24). In the following section each constant is determined after assuming that at very early times the modes are in the Bunch-Davies vacuum, i.e., 


$$
\lim _{k \tau \rightarrow-\infty} U_{s, k}^{+}(\tau) \propto e^{-i k \tau}, \quad \lim _{k \tau \rightarrow-\infty} V_{s, k}^{+}(\tau) \propto e^{i k \tau},
$$

corresponding to the positive and negative frequency solutions, respectively. In addition to that, the amplitudes and the relative phases of the Bunch-Davies vacuum modes are such that the corresponding Hamiltonian is diagonalized.

\section{2. $S_{+}$Hamiltonian}

For $S_{+}$,

$$
\begin{aligned}
H_{+} & =\int \mathrm{d}^{3} k\left(\pi_{\boldsymbol{k}, \alpha}^{\psi+} \partial_{\tau} \psi_{\boldsymbol{k}, \alpha}^{+}\right)-L_{+} \\
& =\int \mathrm{d}^{3} k \psi_{\boldsymbol{k}}^{+, \dagger} \gamma^{0}\left[\gamma^{3} k+\left(2 \xi_{\varphi}-\frac{\xi_{A}}{2}\right) \mathcal{H} \lambda_{4}+\mu_{m} \mathcal{H} \mathrm{I}_{4}\right] \psi_{\boldsymbol{k}}^{+} .
\end{aligned}
$$

Using the mode function expansion from Eqs. (28) and (30) in the Hamiltonian, we arrive at

$$
\begin{aligned}
H_{+}= & \int \mathrm{d}^{3} k \sum_{s= \pm} \frac{1}{2}\left(a_{s, k}^{+\dagger}, b_{s,-k}^{+}\right) \\
& \times\left(\begin{array}{cc}
E\left(u_{s}^{\uparrow}, u_{s}^{\downarrow}\right) & F^{*}\left(u_{s}^{\uparrow, \downarrow}, v_{s}^{\uparrow, \downarrow}\right) \\
F\left(u_{s}^{\uparrow, \downarrow}, v_{s}^{\uparrow, \downarrow}\right) & E\left(v_{s}^{\uparrow}, v_{s}^{\downarrow}\right)
\end{array}\right)\left(\begin{array}{c}
a_{s, k}^{+} \\
b_{s,-k}^{+\dagger}
\end{array}\right),
\end{aligned}
$$

$$
\begin{aligned}
E\left(u_{s}^{\uparrow}, u_{s}^{\downarrow}\right)= & 2\left[k+s\left(2 \xi_{\varphi}-\frac{\xi_{A}}{2}\right) \mathcal{H}\right] \Re\left(u_{s}^{\uparrow *} u_{s}^{\downarrow}\right) \\
& +\mu_{m} \mathcal{H}\left(\left|u_{s}^{\uparrow}\right|^{2}-\left|u_{s}^{\downarrow}\right|^{2}\right), \\
F\left(u_{s}^{\uparrow, \downarrow}, v_{s}^{\uparrow, \downarrow}\right)= & {\left[k+s\left(2 \xi_{\varphi}-\frac{\xi_{A}}{2}\right) \mathcal{H}\right]\left(u_{s}^{\downarrow} v_{s}^{\uparrow *}+u_{s}^{\uparrow} v_{s}^{\downarrow *}\right) } \\
& +\mu_{m} \mathcal{H}\left(v_{s}^{\uparrow *} u_{s}^{\uparrow}-v_{s}^{\downarrow *} u_{s}^{\downarrow}\right) .
\end{aligned}
$$

To bring the Hamiltonian into a diagonal form we make a time-dependent Bogoliubov transformation:

$$
\left(\begin{array}{c}
\check{a}_{s, k}^{+}(\tau) \\
\check{b}_{s,-k}^{+\dagger}(\tau)
\end{array}\right)=\left(\begin{array}{cc}
\alpha_{s, k}(\tau) & \beta_{s, k}(\tau) \\
-\beta_{s, k}^{*}(\tau) & \alpha_{s, k}^{*}(\tau)
\end{array}\right)\left(\begin{array}{c}
a_{s, k}^{+} \\
b_{s,-k}^{+\dagger}
\end{array}\right) .
$$

The new set of time-dependent creation and annihilation operators, $\check{a}_{s, \boldsymbol{k}}^{+}(\tau)$ and $\breve{b}_{s,-\boldsymbol{k}}^{+\dagger}(\tau)$, respect the canonical anticommutation relations, given in Eq. (C3), iff the Bogoliubov coefficients satisfy

$$
\left|\alpha_{s, k}(\tau)\right|^{2}+\left|\beta_{s, k}(\tau)\right|^{2}=1 .
$$

This condition is met and the Hamiltonian is diagonalized as

$$
H_{+}=\int \mathrm{d}^{3} k \sum_{s= \pm}\left[\check{a}_{s, \boldsymbol{k}}^{+\dagger}(\tau) \check{a}_{s, k}^{+}(\tau)-\check{b}_{s,-\boldsymbol{k}}^{+} \check{b}_{s,-\boldsymbol{k}}^{+\dagger}(\tau)\right] \omega_{s, k}(\tau),
$$

where

for

$$
\begin{aligned}
\left|\beta_{s, k}(\tau)\right|^{2} & =\frac{1}{2}\left[1-\frac{E\left(u_{s}^{\uparrow}, u_{s}^{\downarrow}\right)-E\left(v_{s}^{\uparrow}, v_{s}^{\downarrow}\right)}{\sqrt{4\left|F\left(u_{s}^{\uparrow, \downarrow}, v_{s}^{\uparrow, \downarrow}\right)\right|^{2}+\left(E\left(u_{s}^{\uparrow}, u_{s}^{\downarrow}\right)-E\left(v_{s}^{\uparrow}, v_{s}^{\downarrow}\right)\right)^{2}}}\right], \\
\left|\alpha_{s, k}(\tau)\right|^{2} & =\frac{1}{2}\left[1+\frac{E\left(u_{s}^{\uparrow}, u_{s}^{\downarrow}\right)-E\left(v_{s}^{\uparrow}, v_{s}^{\downarrow}\right)}{\sqrt{4\left|F\left(u_{s}^{\uparrow, \downarrow}, v_{s}^{\uparrow, \downarrow}\right)\right|^{2}+\left(E\left(u_{s}^{\uparrow}, u_{s}^{\downarrow}\right)-E\left(v_{s}^{\uparrow}, v_{s}^{\downarrow}\right)\right)^{2}}}\right], \\
\alpha_{s, k}(\tau) & =\left|\alpha_{s, k}(\tau)\right| e^{i \phi_{F}}, \quad \beta_{s, k}(\tau)=\left|\beta_{s, k}(\tau)\right| e^{-i \phi_{F}}, \\
F\left(u_{s}^{\uparrow, \downarrow}, v_{s}^{\uparrow, \downarrow}\right) & =\left|F\left(u_{s}^{\uparrow, \downarrow}, v_{s}^{\uparrow, \downarrow}\right)\right| e^{2 i \phi_{F}} .
\end{aligned}
$$

The effective frequency is given by

$$
\begin{aligned}
\omega_{s, k}(\tau)= & \frac{E\left(u_{s}^{\uparrow}, u_{s}^{\downarrow}\right)+E\left(v_{s}^{\uparrow}, v_{s}^{\downarrow}\right)}{4} \\
& +\frac{1}{4} \sqrt{4\left|F\left(u_{s}^{\uparrow, \downarrow}, v_{s}^{\uparrow, \downarrow}\right)\right|^{2}+\left(E\left(u_{s}^{\uparrow}, u_{s}^{\downarrow}\right)-E\left(v_{s}^{\uparrow}, v_{s}^{\downarrow}\right)\right)^{2}} .
\end{aligned}
$$

It is important to note that the amplitudes and the relative phases of the Bunch-Davies vacuum modes are such that the corresponding Hamiltonian is diagonalized. The following analysis will fix our initial conditions for the positive and negative frequency solutions.

The Bunch-Davies vacuum is defined as

$$
a_{s, k}^{+}\left|0_{B D}\right\rangle=0, \quad b_{s, k}^{+}\left|0_{B D}\right\rangle=0
$$

whereas the instantaneous (or quasiparticle) vacuum as 


$$
\check{a}_{s, k}^{+}(\tau)\left|0_{\tau}\right\rangle=0, \quad \check{b}_{s, k}^{+}(\tau)\left|0_{\tau}\right\rangle=0 .
$$

We work in the Heisenberg picture and we assume that the Universe is in the Bunch-Davies vacuum. The expectation values of observables are calculated with respect to it, i.e., the expected particle number is given by

$$
\begin{aligned}
\check{N}_{s, \boldsymbol{k}}(\tau) & =\left\langle 0_{B D}\left|\check{n}_{s, \boldsymbol{k}}(\tau)\right| 0_{B D}\right\rangle \\
& =\left\langle 0_{B D}\left|\check{a}_{s, \boldsymbol{k}}^{+\dagger}(\tau) \check{a}_{s, \boldsymbol{k}}^{+}(\tau)\right| 0_{B D}\right\rangle=\left|\beta_{s, k}(\tau)\right|^{2} .
\end{aligned}
$$

Hence, $\left|\beta_{s, k}(\tau)\right|^{2}$ is the occupation number of particles with given $s$ and $\boldsymbol{k}$ at a time $\tau$.

We assume that at very early times, $k \tau \rightarrow-\infty, \psi_{k}^{+}$starts in the Bunch-Davies vacuum, i.e., its particle occupation numbers vanish:

$$
\lim _{k \tau \rightarrow-\infty} \beta_{s, k}(\tau)=0
$$

Then it follows from Eq. (C12) that $\lim _{k \tau \rightarrow-\infty} F\left(u_{s}^{\uparrow, \downarrow}\right.$, $\left.v^{\uparrow, \downarrow}\right)=0$, which is satisfied, according to Eq. (C8), if $\lim _{k \tau \rightarrow-\infty}\left(u_{s}^{\uparrow}(k, \tau)-v_{s}^{\uparrow *}(k, \tau)\right)=0$ and $\lim _{k \tau \rightarrow-\infty}\left(u_{s}^{\downarrow}(k, \tau)+\right.$ $\left.v_{s}^{\downarrow *}(k, \tau)\right)=0$. Note that there is some residual freedom in choosing the latter such that the Hamiltonian is diagonalized. One can show that the last two conditions are preserved by the equations of motion, i.e., if imposed initially they hold at later times (for arbitrary $\tau$ ) as well:

$$
u_{s}^{\uparrow}(k, \tau)=v_{s}^{\uparrow *}(k, \tau) \text { and } u_{s}^{\downarrow}(k, \tau)=-v_{s}^{\downarrow *}(k, \tau) .
$$

Equations (C8), (C12), and (C13) then yield (for all $\tau$ )

$$
\begin{aligned}
E\left(u_{s}^{\uparrow}, u_{s}^{\downarrow}\right) & =-E\left(v_{s}^{\uparrow}, v_{s}^{\downarrow}\right), \\
\left|\beta_{s, k}(\tau)\right|^{2} & =\frac{1}{2}\left[1-\frac{E\left(u_{s}^{\uparrow}, u_{s}^{\downarrow}\right)}{2 \omega_{s, k}(\tau)}\right], \\
\left|\alpha_{s, k}(\tau)\right|^{2} & =\frac{1}{2}\left[1+\frac{E\left(u_{s}^{\uparrow}, u_{s}^{\downarrow}\right)}{2 \omega_{s, k}(\tau)}\right],
\end{aligned}
$$

where the effective frequency has been simplified to

$$
\omega_{s, k}(\tau)=\frac{1}{2} \sqrt{|F|^{2}+E\left(u_{s}^{\uparrow}, u_{s}^{\downarrow}\right)^{2}} .
$$

The last condition one has to impose for Eq. (C17) to hold is $\lim _{k \tau \rightarrow-\infty} \Re\left(u_{s}^{\uparrow *}(k, \tau) u_{s}^{\downarrow}(k, \tau)\right)=1$.

Note that then in the Bunch-Davies limit $\lim _{k \tau \rightarrow-\infty} \omega_{s, k}=k$.
Therefore, after applying Eqs. (C4) and (C5) and

$$
\begin{aligned}
& \lim _{k \tau \rightarrow-\infty}\left(u_{s}^{\uparrow}(k, \tau)-v_{s}^{\uparrow *}(k, \tau)\right)=0, \\
& \lim _{k \tau \rightarrow-\infty}\left(u_{s}^{\downarrow}(k, \tau)+v_{s}^{\downarrow *}(k, \tau)\right)=0, \\
& \lim _{k \tau \rightarrow-\infty} \Re\left(u_{s}^{\uparrow *}(k, \tau) u_{s}^{\downarrow}(k, \tau)\right)=1,
\end{aligned}
$$

to Eqs. (36) and (45) we can completely fix the solutions for the $u_{s}^{\uparrow, \downarrow}(k, \tau)$ mode functions, whereas the solutions for $v_{s}^{\uparrow, \downarrow}(k, \tau)$ follow from Eq. (32). We also made use of the asymptotic form of the Whittaker function in (A9).

\section{Quantization of the $S_{-}$fermions}

The quantization prescription for the $\psi_{k}^{-}$modes remains unchanged. It begins with the definition of the canonical conjugate momenta,

$$
\pi_{\boldsymbol{k}, \alpha}^{\psi-}=\frac{\delta S_{-}}{\delta \partial_{\tau} \psi_{\boldsymbol{k}, \alpha}^{-}}=i \psi_{\boldsymbol{k}, \alpha}^{-, *},
$$

where $\alpha$ runs from 1 to 4 . Then $\psi_{\boldsymbol{k}}^{-}$and $\pi_{\boldsymbol{k}}^{\psi-}$ are promoted to quantum operators, satisfying the canonical equal-time anticommutation relations:

$$
\begin{aligned}
& \left\{\psi_{\boldsymbol{k}, \alpha}^{-}(\tau), \psi_{\boldsymbol{k}^{\prime}, \beta}^{-}(\tau)\right\}=0, \\
& \left\{\pi_{\boldsymbol{k}, \alpha}^{\psi-}(\tau), \pi_{\boldsymbol{k}^{\prime}, \beta}^{\psi-}(\tau)\right\}=0, \\
& \left\{\psi_{\boldsymbol{k}, \alpha}^{-}(\tau), \pi_{\boldsymbol{k}^{\prime}, \beta}^{\psi-}(\tau)\right\}=i(2 \pi)^{-3} \delta_{\alpha \beta} \delta^{(3)}\left(\boldsymbol{k}-\boldsymbol{k}^{\prime}\right) .
\end{aligned}
$$

We again postulate that the time-independent coefficients in Eq. (48) are the standard anticommuting creation and annihilation operators, i.e.,

$$
\begin{aligned}
& \left\{a_{s, \boldsymbol{k}}^{-}, a_{s^{\prime}, \boldsymbol{k}^{\prime}}^{-\dagger}\right\}=\delta_{s s^{\prime}} \delta^{(3)}\left(\boldsymbol{k}-\boldsymbol{k}^{\prime}\right), \\
& \left\{b_{s, \boldsymbol{k}}^{-}, b_{s^{\prime}, \boldsymbol{k}^{\prime}}^{-\dagger}\right\}=\delta_{s s^{\prime}} \delta^{(3)}\left(\boldsymbol{k}-\boldsymbol{k}^{\prime}\right),
\end{aligned}
$$

with the rest of the anticommutators vanishing. Equations (C23) and (C24) then imply the normalization condition

$$
\begin{aligned}
& \sum_{s= \pm}\left[\left(U_{s, k}^{-}(\tau)\right)_{\alpha}\left(U_{s, k}^{-\dagger}(\tau)\right)_{\beta}+\left(V_{s, k}^{-}(\tau)\right)_{\alpha}\left(V_{s, k}^{-\dagger}(\tau)\right)_{\beta}\right] \\
& \quad=\delta_{\alpha \beta}(2 \pi)^{-3} .
\end{aligned}
$$

Every term in the square brackets is constant, according to the equation of motion given in Eq. (25). To find the constants, we again assume that the early-time modes are in the Bunch-Davies vacuum,

$$
\lim _{k \tau \rightarrow-\infty} U_{s, k}^{-}(\tau) \propto e^{-i k \tau}, \quad \lim _{k \tau \rightarrow-\infty} V_{s, k}^{-}(\tau) \propto e^{i k \tau} .
$$


Furthermore, the amplitudes and the phases of the modes are adjusted to diagonalize the Hamiltonian.

\section{4. $S_{-}$Hamiltonian}

For $S_{-}$,

$$
\begin{aligned}
H_{-}= & \int \mathrm{d}^{3} k\left(\pi_{\boldsymbol{k}, \alpha}^{\mu-} \partial_{\tau} \psi_{\boldsymbol{k}, \alpha}^{-}\right)-L_{-} \\
= & \int \mathrm{d}^{3} k \psi_{\boldsymbol{k}}^{-, \dagger} \gamma^{0}\left[-\gamma^{3} k-\gamma^{1} \xi_{A} \mathcal{H}+\left(2 \xi_{\varphi}+\frac{\xi_{A}}{2}\right) \mathcal{H} \lambda_{4}\right. \\
& \left.+\mu_{m} \mathcal{H} \mathrm{I}_{4}\right] \psi_{\boldsymbol{k}}^{-} .
\end{aligned}
$$

$$
H_{-}=\int \frac{\mathrm{d}^{3} k}{2}\left(\mathbf{a}_{\boldsymbol{k}}^{-\dagger}, \mathbf{b}_{-\boldsymbol{k}}^{-}\right)\left(\begin{array}{cc}
\mathbf{E}^{u} & \mathbf{F}^{\dagger} \\
\mathbf{F} & \mathbf{E}^{v}
\end{array}\right)\left(\begin{array}{c}
\mathbf{a}_{k}^{-} \\
\left\{b_{-k}^{-\dagger}\right.
\end{array}\right),
$$

where

$$
\begin{aligned}
& \mathbf{a}_{\boldsymbol{k}}^{-}=\left(\begin{array}{c}
a_{+, \boldsymbol{k}}^{-} \\
a_{-, \boldsymbol{k}}^{-}
\end{array}\right), \quad \mathbf{b}_{-\boldsymbol{k}}^{+\dagger}=\left(\begin{array}{c}
b_{+,-\boldsymbol{k}}^{-\dagger} \\
b_{-,-\boldsymbol{k}}^{-\dagger}
\end{array}\right), \\
& \mathbf{E}^{u}=\left(\begin{array}{cc}
E_{+}^{u} & E_{\text {mix }}^{u *} \\
E_{\text {mix }}^{u} & E_{-}^{u}
\end{array}\right), \quad \mathbf{F}=\left(\begin{array}{cc}
F_{+} & F_{-, \text {mix }} \\
F_{+, \text {mix }} & F_{-}
\end{array}\right),
\end{aligned}
$$

and

After using the mode function expansion from Eqs. (48) and (50) in the Hamiltonian, we get

$$
\begin{aligned}
E_{s}^{u}= & \sum_{p= \pm}\left\{-2\left[k+s p\left(2 \xi_{\varphi}+\frac{\xi_{A}}{2}\right) \mathcal{H}\right] \Re\left(u_{s, p}^{\uparrow *} u_{s, p}^{\downarrow}\right)+\mu_{m} \mathcal{H}\left(\left|u_{s, p}^{\uparrow}\right|^{2}-\left|u_{s, p}^{\downarrow}\right|^{2}\right)+2 s p \xi_{A} \mathcal{H} \Re\left(u_{s, p}^{\uparrow *} u_{s,-p}^{\downarrow}\right)\right\}, \\
F_{s}= & \sum_{p= \pm}\left\{-\left[k+s p\left(2 \xi_{\varphi}+\frac{\xi_{A}}{2}\right) \mathcal{H}\right]\left(v_{s, p}^{\uparrow *} u_{s, p}^{\downarrow}+v_{s, p}^{\downarrow *} u_{s, p}^{\uparrow}\right)+\mu_{m} \mathcal{H}\left(v_{s, p}^{\uparrow *} u_{s, p}^{\uparrow}-v_{s, p}^{\downarrow *} u_{s, p}^{\downarrow}\right)+s p \xi_{A} \mathcal{H}\left(v_{s, p}^{\uparrow *} u_{s,-p}^{\downarrow}+u_{s, p}^{\uparrow} v_{s,-p}^{\downarrow *}\right)\right\}, \\
E_{\text {mix }}^{u}= & \sum_{p= \pm}\left\{-\left[k+p\left(2 \xi_{\varphi}+\frac{\xi_{A}}{2}\right) \mathcal{H}\right]\left(u_{-,-p}^{\uparrow *} u_{+, p}^{\downarrow}+u_{-,-p}^{\downarrow *} u_{+, p}^{\uparrow}\right)+\mu_{m} \mathcal{H}\left(u_{-,-p}^{\uparrow *} u_{+, p}^{\uparrow}-u_{-,-p}^{\downarrow *} u_{+, p}^{\downarrow}\right)\right. \\
& \left.+p \xi_{A} \mathcal{H}\left(u_{-,-p}^{\uparrow *} u_{+,-p}^{\downarrow}+u_{+, p}^{\uparrow} u_{-, p}^{\downarrow *}\right)\right\}, \\
F_{s, \text { mix }}= & \sum_{p= \pm}\left\{-\left[k+s p\left(2 \xi_{\varphi}+\frac{\xi_{A}}{2}\right) \mathcal{H}\right]\left(v_{-s,-p}^{\uparrow *} u_{s, p}^{\downarrow}+v_{-s,-p}^{\downarrow *} u_{s, p}^{\uparrow}\right)+\mu_{m} \mathcal{H}\left(v_{-s, p}^{\uparrow *} u_{s,-p}^{\uparrow}-v_{-s, p}^{\downarrow *} u_{s,-p}^{\downarrow}\right)\right. \\
& \left.+s p \xi_{A} \mathcal{H}\left(v_{-s,-p}^{\uparrow *} u_{s,-p}^{\downarrow}+u_{s, p}^{\uparrow} v_{-s, p}^{\downarrow *}\right)\right\} .
\end{aligned}
$$

The Hamiltonian can be diagonalized after making a timedependent Bogoliubov transformation,

$$
\left(\begin{array}{c}
\check{\mathbf{a}}_{k}^{-}(\tau) \\
\check{\mathbf{b}}_{-\boldsymbol{k}}^{-\dagger}(\tau)
\end{array}\right)=P_{k}(\tau)\left(\begin{array}{c}
\mathbf{a}_{k}^{-} \\
b_{-k}^{-\dagger}
\end{array}\right) .
$$

The time-dependent creation and annihilation operators, $\check{\mathbf{a}}_{\boldsymbol{k}}^{-}(\tau)$ and $\check{\mathbf{b}}_{-\boldsymbol{k}}^{-\dagger}(\tau)$, obey the canonical anticommutation relations from Eq. (C24), iff the transformation matrix is unitary:

$$
P_{k}(\tau) P_{k}^{\dagger}(\tau)=\mathrm{I}_{4} .
$$

We then impose that

$$
P_{k}(\tau)\left(\begin{array}{cc}
\mathbf{E}^{u} & \mathbf{F}^{\dagger} \\
\mathbf{F} & \mathbf{E}^{v}
\end{array}\right) P_{k}^{\dagger}(\tau)
$$

is diagonal, implying that the eigenvectors of $\left(\begin{array}{cc}\mathbf{E}^{u} & \mathbf{F}^{\dagger} \\ \mathbf{F} & \mathbf{E}^{v}\end{array}\right)$ are the columns of $P_{k}^{\dagger}(\tau)$.
Due to the nature of the equations of motion in the $S_{-}$ we cannot have analytical expressions for the effective frequency $\omega_{s, k}^{-}(\tau)$ as opposed to Eq. (C13) in $S_{+}$. Therefore, the expectation values of observables are calculated numerically.

At early times all off-diagonal terms of the Hamiltonian should vanish for both $s= \pm$. In other words $P_{k}(\tau)=\mathbf{I}_{4}$, which is equivalent to having

$$
H_{-}=\left(\begin{array}{cc}
\mathbf{I}_{2} & 0 \\
0 & -\mathbf{I}_{2}
\end{array}\right)
$$

It follows from Eq. (C30) that

$\lim _{k \tau \rightarrow-\infty} F_{s}=0, \quad \lim _{k \tau \rightarrow-\infty} E_{\text {mix }}^{u}=0, \quad \lim _{k \tau \rightarrow-\infty} F_{s, \text { mix }}=0$.

The above conditions are met, if the following equations are satisfied: 
(1) $\lim _{k \tau \rightarrow-\infty}\left(u_{s,+}^{\uparrow}(k, \tau)-v_{s,+}^{\uparrow *}(k, \tau)\right)=0, \quad \lim _{k \tau \rightarrow-\infty}$ $\left(u_{s,+}^{\downarrow}(k, \tau)+v_{s,+}^{\downarrow *}(k, \tau)\right)=0$.

It can be shown that these conditions are preserved by the equations of motion, so if imposed once they hold for any arbitrary $\tau$ as well

$$
u_{s,+}^{\uparrow}(k, \tau)=v_{s,+}^{\uparrow *}(k, \tau), u_{s,+}^{\downarrow}(k, \tau)=-v_{s,+}^{\downarrow *}(k, \tau) .
$$

(2) $\lim _{k \tau \rightarrow-\infty} \Re\left(u_{s,+}^{\uparrow *}(k, \tau) u_{s,+}^{\downarrow}(k, \tau)\right)=-1 \quad$ and $\lim _{k \tau \rightarrow-\infty} \Re\left(v_{s,+}^{\uparrow *}(k, \tau) v_{s,+}^{\downarrow}(k, \tau)\right)=1$. This condition comes from Eq. (C34).

(3) $\lim _{k \tau \rightarrow-\infty} u_{s,-}^{\uparrow}(k, \tau)=0$ and $\lim _{k \tau \rightarrow-\infty} v_{s,-}^{\uparrow, \downarrow}(k, \tau)=0$. This condition is imposed so that the particle occupation number vanishes at early times. Note that this is an arbitrary choice and one can make the mode functions vanish for, e.g., $p=+$.

Using the above we can solve the equations of motion numerically.
[1] A. Maleknejad and M. M. Sheikh-Jabbari, Gauge-flation: Inflation from non-Abelian gauge fields, Phys. Lett. B 723, 224 (2013).

[2] A. Maleknejad and M. M. Sheikh-Jabbari, Non-Abelian gauge field inflation, Phys. Rev. D 84, 043515 (2011).

[3] P. Adshead and M. Wyman, Chromo-Natural Inflation: Natural Inflation on a Steep Potential with Classical NonAbelian Gauge Fields, Phys. Rev. Lett. 108, 261302 (2012).

[4] P. Adshead, E. Martinec, and M. Wyman, Perturbations in chromo-natural inflation, J. High Energy Phys. 09 (2013) 087.

[5] A. H. Guth, The inflationary Universe: A possible solution to the horizon and flatness problems, Phys. Rev. D 23, 347 (1981).

[6] K. Sato, First order phase transition of a vacuum and expansion of the Universe, Mon. Not. R. Astron. Soc. 195, 467 (1981).

[7] A. D. Linde, A new inflationary Universe scenario: A possible solution of the horizon, flatness, homogeneity, isotropy and primordial monopole problems, Phys. Lett. 108B, 389 (1982).

[8] A. Albrecht and P. J. Steinhardt, Cosmology for Grand Unified Theories with Radiatively Induced Symmetry Breaking, Phys. Rev. Lett. 48, 1220 (1982).

[9] A. Maleknejad, M. M. Sheikh-Jabbari, and J. Soda, Gauge fields and inflation, Phys. Rep. 528, 161 (2013).

[10] A. Maleknejad, Axion inflation with an SU(2) gauge field: Detectable chiral gravity waves, J. High Energy Phys. 07 (2016) 104.

[11] P. Adshead, E. Martinec, E. I. Sfakianakis, and M. Wyman, Higgsed chromo-natural inflation, J. High Energy Phys. 12 (2016) 137.

[12] E. Dimastrogiovanni, M. Fasiello, and T. Fujita, Primordial gravitational waves from axion-gauge fields dynamics, J. Cosmol. Astropart. Phys. 01 (2017) 019.

[13] P. Adshead and E. I. Sfakianakis, Higgsed gauge-flation, J. High Energy Phys. 08 (2017) 130.

[14] E. Dimastrogiovanni and M. Peloso, Stability analysis of chromo-natural inflation and possible evasion of Lyth's bound, Phys. Rev. D 87, 103501 (2013).

[15] E. Dimastrogiovanni, M. Fasiello, and A. J. Tolley, Lowenergy effective field theory for chromo-natural inflation, J. Cosmol. Astropart. Phys. 02 (2013) 046.
[16] P. Adshead, E. Martinec, and M. Wyman, Gauge fields and inflation: Chiral gravitational waves, fluctuations, and the Lyth bound, Phys. Rev. D 88, 021302 (2013).

[17] A. Agrawal, T. Fujita, and E. Komatsu, Large tensor nonGaussianity from axion-gauge fields dynamics, Phys. Rev. D 97, 103526 (2018).

[18] A. Agrawal, T. Fujita, and E. Komatsu, Tensor nonGaussianity from axion-gauge-fields dynamics: Parameter search, J. Cosmol. Astropart. Phys. 06 (2018) 027.

[19] E. Dimastrogiovanni, M. Fasiello, R. J. Hardwick, H. Assadullahi, K. Koyama, and D. Wands, Non-Gaussianity from axion-gauge fields interactions during inflation, J. Cosmol. Astropart. Phys. 11 (2018) 029.

[20] A. Maleknejad, Gravitational leptogenesis in axion inflation with SU(2) gauge field, J. Cosmol. Astropart. Phys. 12 (2016) 027.

[21] P. Adshead, A. J. Long, and E. I. Sfakianakis, Gravitational leptogenesis, reheating, and models of neutrino mass, Phys. Rev. D 97, 043511 (2018).

[22] R. R. Caldwell and C. Devulder, Axion gauge field inflation and gravitational leptogenesis: A lower bound on B modes from the matter-antimatter asymmetry of the Universe, Phys. Rev. D 97, 023532 (2018).

[23] P. Adshead, J. T. Giblin, and Z. J. Weiner, Gravitational waves from gauge preheating, Phys. Rev. D 98, 043525 (2018).

[24] A. Papageorgiou, M. Peloso, and C. Unal, Nonlinear perturbations from the coupling of the inflaton to a nonAbelian gauge field, with a focus on chromo-natural inflation, J. Cosmol. Astropart. Phys. 09 (2018) 030.

[25] A. Papageorgiou, M. Peloso, and C. Unal, Nonlinear perturbations from axion-gauge fields dynamics during inflation, J. Cosmol. Astropart. Phys. 07 (2019) 004.

[26] T. Matsumura, Y. Akiba, J. Borrill, Y. Chinone, M. Dobbs, H. Fuke et al., Mission design of LiteBIRD, J. Low Temp. Phys. 176, 733 (2014).

[27] M. Hazumi et al., LiteBIRD: A satellite for the studies of B-mode polarization and inflation from cosmic background radiation detection, J. Low Temp. Phys. 194, 443 (2019).

[28] CMB-S4 Collaboration, CMB-S4 Science Book, First Edition, arXiv:1610.02743. 
[29] B. Thorne, T. Fujita, M. Hazumi, N. Katayama, E. Komatsu, and M. Shiraishi, Finding the chiral gravitational wave background of an axion-SU(2) inflationary model using CMB observations and laser interferometers, Phys. Rev. D 97, 043506 (2018).

[30] S. Shandera et al., Probing the origin of our Universe through cosmic microwave background constraints on gravitational waves, Bull. Am. Astron. Soc. 51, 338 (2019).

[31] K. D. Lozanov, A. Maleknejad, and E. Komatsu, Schwinger effect by an $S U(2)$ gauge field during inflation, J. High Energy Phys. 02 (2019) 041.

[32] J. Schwinger, On gauge invariance and vacuum polarization, Phys. Rev. 82, 664 (1951).

[33] A. Maleknejad and E. Komatsu, Production and backreaction of spin-2 particles of $S U(2)$ gauge field during inflation, J. High Energy Phys. 05 (2019) 174.

[34] T. Hayashinaka, T. Fujita, and J. Yokoyama, Fermionic Schwinger effect and induced current in de Sitter space, J. Cosmol. Astropart. Phys. 07 (2016) 010.

[35] P. Adshead and E. I. Sfakianakis, Fermion production during and after axion inflation, J. Cosmol. Astropart. Phys. 11 (2015) 021.

[36] P. Adshead, L. Pearce, M. Peloso, M. A. Roberts, and L. Sorbo, Phenomenology of fermion production during axion inflation, J. Cosmol. Astropart. Phys. 06 (2018) 020.

[37] V. Domcke and K. Mukaida, Gauge field and fermion production during axion inflation, J. Cosmol. Astropart. Phys. 11 (2018) 020.
[38] V. Domcke, Y. Ema, K. Mukaida, and R. Sato, Chiral anomaly and Schwinger effect in non-Abelian gauge theories, J. High Energy Phys. 03 (2019) 111.

[39] M. B. Frob, J. Garriga, S. Kanno, M. Sasaki, J. Soda, T. Tanaka, and A. Vilenkin, Schwinger effect in de Sitter space, J. Cosmol. Astropart. Phys. 04 (2014) 009.

[40] T. Kobayashi and N. Afshordi, Schwinger effect in 4D de Sitter space and constraints on magnetogenesis in the early Universe, J. High Energy Phys. 10 (2014) 166.

[41] R. Sharma and S. Singh, Multifaceted Schwinger effect in de Sitter space, Phys. Rev. D 96, 025012 (2017).

[42] H. Kitamoto, Schwinger effect in inflaton-driven electric field, Phys. Rev. D 98, 103512 (2018).

[43] S. Shakeri, M. A. Gorji, and H. Firouzjahi, Schwinger mechanism during inflation, Phys. Rev. D 99, 103525 (2019).

[44] S. Weinberg, The Quantum Theory of Fields. Vol. 2: Modern Applications (Cambridge University Press, Cambridge, England, 2013).

[45] L. Parker and D. Toms, Quantum Field Theory in Curved Spacetime: Quantized Fields and Gravity, Cambridge Monographs on Mathematical Physics (Cambridge University Press, Cambridge, England, 2009).

[46] D. G. Figueroa and T. Meriniemi, Stochastic background of gravitational waves from fermions-Theory and applications, J. High Energy Phys. 10 (2013) 101.

[47] A. Maleknejad, Dark fermions and spontaneous $C P$ violation in $S U(2)$-axion inflation, arXiv:1909.11545.

[48] Planck Collaboration, Planck 2015 results. XIII. Cosmological parameters, Astron. Astrophys. 594, A13 (2016). 